CERN-PPE/95-102

July 4th, 1995

\title{
Inclusive Charged Particle Cross Sections in Full Phase Space from Proton Proton Interactions at ISR Energies
}

\author{
Ames-Bologna-CERN-Dortmund-Heidelberg-Warsaw Collaboration
}

\begin{abstract}
Measurements are presented of inclusive charged particle production in proton-proton collisions at $\sqrt{s}=31,44$ and $62 \mathrm{GeV}$ at the CERN ISR. The large acceptance of the Split Field Magnet detector allows a single experimental setup to cover almost the full longitudinal phase space up to a transverse momentum of $3 \mathrm{GeV} / \mathrm{c}$.
\end{abstract}

(Submitted to Z. Phys. C Particles and Fields)

* See the following page for the list of authors. 


\section{Ames-Bologna-CERN-Dortmund-Heidelberg-Warsaw Collaboration}

A. Breakstone ${ }^{1}$, H.B. Crawley, A. Firestone, M. Gorbics ${ }^{2}$, L.D. Isenhower ${ }^{3}$, J.W. Lamsa, W.T. Meyer

Ames Laboratory and Physics Department, lowa State University, Ames IA 50011, USA ${ }^{14}$

R. Campanini, M. Cuffiani, G.M. Dallavalle, M.M. Deninno, F. Fabbri, G. Giacomelli, F. Rimondi, G.P. Siroli

Dipartimento di Fisica dell' Universita di Bologna and INFN, 40126 Bologna, Italy

D. Drijard, H.G. Fischer, W. Geist ${ }^{4}$, G. Mornacchi, M. Panter ${ }^{5}$, O. Ullaland, R. Yeung ${ }^{6}$

European Laboratory for Particle Physics (CERN), 1211 Geneva 23, Switzerland

T. Lohse ${ }^{7}$, R. Mankel $^{7}$, K. Rauschnabel ${ }^{8}$, M. Schmelling ${ }^{9}$, D. Wegener

Institut für Physik der Universität Dortmund, 44227 Dortmund, Germany ${ }^{15}$

H. Flöge, H. Frehse ${ }^{10}$, L. Gesswein, P. Hanke, M. Heiden ${ }^{11}$, W. Herr ${ }^{12}$, E.E. Kluge, T. Nakada ${ }^{13}$, A. Putzer,

B. Rensch

Institut für Hochenergiephysik, Universität Heidelberg, 69120 Heidelberg, Germany ${ }^{15}$

K. Doroba, R. Gokieli, R. Sosnowski, M. Szczekowski

Institute for Nuclear Studies and University of Warsaw, 00681 Warsaw, Poland

\footnotetext{
${ }^{1}$ Now at University of Hawaii, Honolulu HI 96822, USA.

${ }^{2}$ Now at LeCroy Research Systems, Chestnut Ridge, New York, USA.

${ }^{3}$ Now at Abilene Christian University, Abilene TX 79669, USA.

${ }^{4}$ Now at CRN-IN2P3/ULP, 67037 Strasbourg, France.

${ }^{5}$ Now at MPI für Kernphysik, 69029 Heidelberg, Germany.

${ }^{6}$ Now at CALTECH, Pasadena CA 91125, USA.

${ }^{7}$ Now at Humboldt Universität, 10115 Berlin, Germany.

${ }^{8}$ Now at Fachhochschule für Technik, 74080 Heilbronn, Germany.

${ }^{9}$ Now at CERN, PPE Division, 1211 Geneva 23, Switzerland.

${ }^{10}$ Now at CONVEX, 8000 Zürich, Switzerland.

${ }^{11}$ Now at DEC, 85774 Unterföhring, Germany.

${ }^{12}$ Now at CERN, SL Division, 1211 Geneva 23, Switzerland.

${ }^{13}$ Now at PSI, 5232 Villigen, Switzerland.

${ }^{14}$ Supported by the U.S. Department of Energy under contract W-7405-ENG-82.

${ }^{15}$ Supported by the Bundesministerium für Forschung und Technologie, Germany.
} 


\section{Introduction}

Our present understanding of hadronic collisions is still based to a large extent on phenomenology: the bulk of the total interaction cross section falls into the non-perturbative sector of quantum chromodynamics and is thus not accessible to precise calculation.

It is only in some small regions of the total available phase space that perturbative QCD on the parton level has been applied with good success. This is the case e.g. for single particle [1] and jet [2] production at large transverse momentum and for certain aspects of elastic [3] and diffractive [4] scattering, especially for single and double [5] Pomeron exchange.

The linking of these sectors to the majority of "soft" collisions, the interpolation between different center-of-mass energies and especially the extrapolation to the upcoming higher interaction energies have therefore had to rely on a multitude of phenomenological methods, such as those based on multiperipheral cluster models [6],[7], uncorrelated jet models [8], or string models [9]. This whole field has gained considerable interest with the search for a new state of hadronic matter in ultrarelativistic heavy ion collisions [10], where one has to rely heavily on a precise knowledge of the underlying elementary hadron-hadron interaction.

The evaluation of relative precision and predictive power of the available phenomenological approaches requires a comparison with data. These data should have good precision and internal consistency and should, in particular, allow for good coverage of the total phase space.

It is in this spirit that we present for the first time a complete set of inclusive charged hadron cross sections in the energy range of the CERN Intersecting Storage Rings, $30 \mathrm{GeV}<\sqrt{s}<62$ GeV. These data are from the Split Field Magnet detector and cover the total available range of rapidities for transverse momenta up to $3 \mathrm{GeV} / \mathrm{c}$. They therefore span the transition region from soft to hard hadronic collisions and a good part of the region of high transverse momenta which has been shown [1] to be dominated by hard processes.

\section{Data acquisition and event reconstruction}

The experiment was performed at the CERN Intersecting Storage Rings (ISR) using the Split Field Magnet (SFM) detector, which had a maximum field strength of $1.0 \mathrm{~T}$. The magnetic volume of the detector was filled with Multiwire Proportional Chambers, which measured the momenta of charged particles in nearly the full solid angle. Details on the detector can be found elsewhere [11]. The experiment used a minimum bias trigger, which essentially required the presence of at least one charged track in the detector. This trigger accepted $95 \%$ of the inelastic cross section.

The data were taken at three different c.m. energies. About 900,000 events are used at $\sqrt{s}$ $=62 \mathrm{GeV}, 1,000,000$ events at $\sqrt{s}=44 \mathrm{GeV}$, and 1,500,000 events at $\sqrt{s}=31 \mathrm{GeV}$.

These events were processed through the standard SFM off-line analysis programs. All particles were assumed to be pions because particle identification was limited to a small region of phase space.

About $80 \%$ of the charged tracks are measured with a relative momentum precision $\delta p / p<10 \%$. Careful analysis of the data has shown that the corrected cross sections do not significantly depend on the precise cut-off in $\delta p / p$ for $\delta p / p<30 \%$. For this analysis only tracks with $\delta p / p<10 \%$ have been retained. 


\section{Acceptance corrections and systematic errors}

Acceptance corrections were calculated using a Monte-Carlo technique. Charged particles were generated in full phase space according to $\frac{d^{3} \sigma}{d \phi d y d p_{T}^{2}}=C \cdot e^{-6 p_{T}}$, where $\phi$ is the azimuthal angle and $y$ the cms rapidity.

The response of the detector was simulated as these particles were tracked through the magnetic field. The Monte-Carlo program included energy loss, secondary interaction processes, and particle decays. To obtain acceptance tables, the generated particles were passed through the same reconstruction program as used for real events.

As the acceptance is strongly varying in some regions of phase space, a fine binning was chosen for the evaluation of acceptance corrections:

$$
\begin{aligned}
& \Delta|y|=0.5 \quad \text { for all rapidities, } \\
& \Delta \phi=18^{\circ} \quad \text { for all azimuthal angles, } \\
& \Delta p_{T}=0.1 \mathrm{GeV} / \mathrm{c} \text { for } 0.2 \mathrm{GeV} / \mathrm{c}<p_{T}<0.7 \mathrm{GeV} / \mathrm{c} \text {, } \\
& \Delta p_{T}=0.3 \mathrm{GeV} / \mathrm{c} \text { for } 0.7 \mathrm{GeV} / \mathrm{c}<p_{T}<1.9 \mathrm{GeV} / \mathrm{c} \text {, and } \\
& \Delta p_{T}=0.8 \mathrm{GeV} / \mathrm{c} \text { for } 1.9 \mathrm{GeV} / \mathrm{c}<p_{T}<3.5 \mathrm{GeV} / \mathrm{c} \text {. }
\end{aligned}
$$

The acceptance is given by $\epsilon=n_{\text {rec }} / n_{\text {gen }}$ where $n_{\text {rec }}$ is the number of tracks with reconstructed momenta inside a given bin and $n_{\text {gen }}$ is the number of tracks generated with a momentum inside this bin. The error of the acceptance is $\Delta \epsilon / \epsilon=\sqrt{\left(\left|1 / n_{\text {rec }}-1 / n_{\text {gen }}\right|\right)}$,

To obtain absolute cross sections the integrated luminosity corresponding to the data sample is needed. This has been done using three different scintillation counter monitor systems, calibrated by the van der Meer method [12].

The systematic error comes from the error on the luminosity measurement, from the accuracy in the acceptance determination, and from the effects of $V^{0} \mathrm{~s}$ simulating primary tracks. It is estimated to be between $6 \%$ (at $\mathrm{y}=0$ ) and $10 \%$ (at maximum rapidity).

\section{Experimental results}

The cross section in each bin of phase space is calculated according to:

$$
E \cdot \frac{d^{3} \sigma}{d p^{3}}=\frac{c_{m o n}}{N} \cdot \frac{n}{2 \pi \cdot \Delta \phi \cdot \Delta|y| \cdot \Delta p_{T}} \cdot \frac{1}{p_{T}} \cdot \frac{1}{\epsilon}
$$

where $c_{\text {mon }}$ is the normalization constant obtained from the monitor counters, $N$ is the total number of events used, $n$ is the number of tracks per bin, and $\epsilon$ is the acceptance. The acceptance corrected cross section was integrated over $\phi$. The final bin sizes have been chosen to keep the statistical errors smaller than the systematic ones.

It is worth noting that systematic errors influence mainly the overall normalization, whereas the uncertainty of the shape of individual distributions is dominated by statistical errors.

For low $p_{T}$ bins, the statistical errors are always below $0.1 \%$; only for $p_{T}>2 \mathrm{GeV} / \mathrm{c}$ do they approach a maximum value of about $5 \%$. For the remaining phase space, the errors on the cross sections are between 1 and $2 \%$ [13].

The measured cross sections are presented as a function of $y$ for different $p_{T}$ values (tables 1-3). As no particle identification was used, the pion mass is assumed for the transformation into the overall c.m. system. The fraction of heavier particles, such as kaons and protons, depends on the phase space region, and this may distort the distributions. The experimental 
measurements are therefore also given as a function of the Feynman variable, $x_{F}$, since this variable is independent of the mass assignment (tables 4-6).

\subsection{Inclusive cross sections as a function of transverse momentum}

Fig. 1 shows the differential cross section of charged particles measured at $\sqrt{s}=62 \mathrm{GeV}$ as a function of $p_{T}$ at a fixed value of the rapidity, $\langle|y|\rangle=0.75$. Also given are previous measurements of charged pions performed by this collaboration with a dedicated trigger on high transverse momentum [14]. At low $p_{T}$, an approximately exponential behavior is found as expected from the scattering of extended objects. With increasing $p_{T}$ one observes a weaker dependence on $p_{T}$, which is reproduced qualitatively in the framework of perturbative QCD.

Differential cross sections are presented in figures $2 \mathrm{a}$ and $2 \mathrm{~b}$ for two different rapidity values, chosen to permit comparison with data from other experiments [15], [16]. In both cases, differential cross sections are shown for three energies $(\sqrt{s}=62 \mathrm{GeV}, 44 \mathrm{GeV}$, and $31 \mathrm{GeV})$, and for both charges, scaled by factors of 10 for clarity. From the top to the bottom of the figure $\sqrt{s}$ decreases, and the cross sections for positive particles are always above the ones for negative particles. Fig. 2a contains also data from the British-Scandinavian (BS) Collaboration [15]taken at the corresponding energies. They are in good agreement with the present measurements. Fig. $2 \mathrm{~b}$ includes data from the CCHK collaboration [16] at $\sqrt{s}=52.5 \mathrm{GeV}$, which tend to be compatible with the present data at $\sqrt{s}=62 \mathrm{GeV}$.

\subsection{Inclusive cross sections as a function of rapidity}

Differential cross sections as a function of rapidity are given for eleven $p_{T}$ intervals at $\sqrt{s}=62$ $\mathrm{GeV}$, separately for positive particles in fig. 3a and negative particles in fig. $3 \mathrm{~b}$. Both figures cover the entire longitudinal phase space. At low $p_{T}$, a rapidity plateau is seen, which extends further for positive than for negative particles. Due mainly to the large fraction of protons at high rapidity, the decrease of the cross section is weaker for positive than for negative particles in this region of phase space.

Figure 4 shows the invariant cross sections at central rapidity as a function of $\sqrt{s}$ for different $p_{T}$ values. The increase in height of the central rapidity plateau as a function of energy is clearly visible for both charges.

It is interesting to test if the change in the shape of rapidity distributions as a function of $p_{T}$ and $\sqrt{s}$ is nothing but a consequence of momentum conservation. Lorentz-invariant differential cross sections are determined as functions of the variable $\xi=y / y_{\max }\left(m, p_{T}\right)$ where $y_{\max }\left(m, p_{T}\right)$ is the kinematic limit for production of secondary hadrons of mass $m$ with transverse momentum $p_{T}$ in pp collisions at $\sqrt{s}$. Figure 5 shows the experimental distribution for negative particles as a function of $\xi$ using the pion mass. Negative particles were selected as they are not present in the initial state of the scattering process and thus are a direct probe of the underlying particle production mechanism. One finds that for eight intervals of $p_{T}$ at given $\sqrt{s}$ the shapes of all distributions are compatible with each other. In addition, it seems that the shapes are not a strong function of $\sqrt{s}$. 


\subsection{Parametrization of inclusive cross sections}

The dependence of the cross sections on $\sqrt{s}, y$ and $p_{T}$ can be parametrized by the following ansatz,

$$
\sigma=\exp \left(\sum_{k l} a_{k l} p_{T}^{k} z^{l}\right) \quad \text { with } z=\left(\frac{y}{\ln \left(\sqrt{s} / m_{\pi}\right)}\right)^{2} \quad \text { and } \quad a_{k l}=A_{k l}\left(\frac{\sqrt{s}}{2 m_{p}}\right)^{P_{k l}}
$$

Here $p_{T}$ is measured in units of $\mathrm{GeV} / c$ and $m_{\pi}$ and $m_{p}$ denote the pion and the proton mass, respectively. Only the leading order coefficients $a_{k l}$ are needed to obtain a good description of the experimental data. The fitted values $A_{k l}$ and $P_{k l}$ are given in the following table.

\begin{tabular}{|r|r|r|r|r|}
\hline \multirow{2}{*}{} & \multicolumn{2}{|c|}{ Positives } & \multicolumn{2}{c|}{ Negatives } \\
\cline { 2 - 5 }$A_{k l}$ & \multicolumn{1}{|c|}{$P_{k l}$} & \multicolumn{1}{c|}{$A_{k l}$} & \multicolumn{1}{c|}{$P_{k l}$} \\
\hline$a_{00}$ & 4.8164 & 0.0321 & 5.4505 & 0.0010 \\
$a_{10}$ & -6.2953 & 0.0129 & -9.5992 & -0.0872 \\
$a_{20}$ & 0.3332 & 0.3755 & 2.9395 & -0.2122 \\
$a_{30}$ & -0.0155 & 0.6649 & -1.3642 & -0.6201 \\
\hline$a_{01}$ & -5.1510 & 0.0079 & -14.2117 & -0.2962 \\
$a_{11}$ & 11.0073 & -0.0046 & 36.6920 & -0.2304 \\
$a_{21}$ & -5.3261 & 0.1999 & -80.1132 & -0.4826 \\
$a_{31}$ & 0.5068 & 0.4258 & 64.8912 & -0.9479 \\
\hline$a_{12}$ & & & -38.2022 & 0.0793 \\
$a_{22}$ & & & 5.8091 & 0.4283 \\
\hline
\end{tabular}

The parameters for each charge were determined simultaneously by a global $\chi^{2}$ fit. In order to take binning effects properly into account, each data point was compared to the average function value over its $y-p_{T}$ bin. The data deviate from the parametrization by less than $10 \%$, consistent with the estimates of the systematic uncertainties. Figures $6 \mathrm{a}-\mathrm{f}$ show the measured invariant cross sections together with this parametrization.

\section{Conclusions}

Invariant differential cross sections of charged particles produced in proton proton interactions at ISR energies have been measured over the full phase space. As all data have been taken with an identical experimental setup, most of the systematic errors cancel when taking ratios. This and the high statistics available allow for the first time an almost unbiased study of the dependence of the invariant cross section on kinematical variables and collision energy.

\section{Acknowledgement}

As this is likely to be the last in a long series of more than 80 publications by this collaboration and its predecessors based upon measurements of $\mathrm{pp}, \bar{p} \mathrm{p}, \alpha \mathrm{p}$ and $\alpha \alpha$ collisions with the SFM detector we would like to thank CERN and its various divisions and technical staff for support over more than 20 years, starting in 1974, as well as our former colleagues for sharing with us their knowledge and enthusiasm. Those of us from non-member countries thank CERN for its hospitality. 


\section{References}

[1] W.M. Geist et al., Phys. Rep. 197 (1990) 263

[2] K. Jakobs, Int. Journ. Mod. Phys. A9 (1994) 2903

[3] A. Martin, Nucl. Phys. B (Proc. Suppl.) 12 (1990) 1

[4] P.V. Landshoff, Nucl. Phys. B (Proc. Suppl.) 12 (1990) 397

[5] A. Breakstone et al., " Exploring the Pomeron:

Transverse Momentum Distributions in Pomeron-Pomeron Collisions at the CERN ISR", CERN/EP/3240R (1986)

[6] M. LeBellac, "Short Range Order and Local Conservation of Quantum Numbers in Multiparticle Production", CERN 76-14 (1976)

[7] M. LeBellac, Proc. of the Europ. Conf. on Particle Physics, Budapest(1977) 411

[8] E.H. de Groot, H. Satz, Nucl. Phys. B 130 (1977) 257

[9] A. Capella et. al. Phys. Rep. 236 (1994), 225

[10] Proc. of the $10^{\text {th }}$ Int. Conf. on Ultrarelativistic Nucleus-Nucleus Collisions, Borlänge, Sweden, 1933 (Quark Matter 93) Nucl. Phys. A 566 (1994)

[11] R. Bouclier et al., Nucl. Inst. and Meth. 125 (1975) 19

[12] S. van der Meer, CERN Internal Report ISR - PO/68 - 31

[13] H. Flöge, Diploma Thesis, Heidelberg (1987)

[14] D. Drijard et al.,CDHW-Collaboration, Nucl. Phys. B 208 (1982) 1

[15] B. Alper et al., BS-Collaboration, Nucl. Phys. B 87 (1975) 19

[16] R. Cottrell et al., CCHK-Collaboration, Phys. Lett. 55 B (1975) 341 


\section{Table Captions}

Tab. 1. Invariant cross sections for positive $(\oplus)$ and negative $(\ominus)$ particles at $\sqrt{s}=62 \mathrm{GeV}$ as functions of $y$ for different $p_{T}$ values.

Tab. 2. Invariant cross sections for positive $(\oplus)$ and negative $(\ominus)$ particles at $\sqrt{s}=44 \mathrm{GeV}$ as functions of $y$ for different $p_{T}$ values.

Tab. 3. Invariant cross sections for positive $(\oplus)$ and negative $(\ominus)$ particles at $\sqrt{s}=31 \mathrm{GeV}$ as functions of $y$ for different $p_{T}$ values.

Tab. 4. Invariant cross sections for positive $(\oplus)$ and negative $(\ominus)$ particles at $\sqrt{s}=62 \mathrm{GeV}$ as functions of $x_{F}$ for different $p_{T}$ values.

Tab. 5. Invariant cross sections for positive $(\oplus)$ and negative $(\ominus)$ particles at $\sqrt{s}=44 \mathrm{GeV}$ as functions of $x_{F}$ for different $p_{T}$ values.

Tab. 6. Invariant cross sections for positive $(\oplus)$ and negative $(\ominus)$ particles at $\sqrt{s}=31 \mathrm{GeV}$ as functions of $x_{F}$ for different $p_{T}$ values. 


\section{Figure Captions}

Fig. 1. Invariant cross sections as functions of $p_{T}$ at $\langle|y|\rangle=0.75$. The cross sections for positive particles (circles) are multiplied by a factor of 10 for better distinction from those of negative ones (squares). Also shown are the high $p_{T}$ data from CDHW [14] for $4<p_{T}<12$ $\mathrm{GeV} / \mathrm{c}$. The line corresponds to a fit to the negative cross section for $p_{T} \leq 0.7 \mathrm{GeV} / \mathrm{c}$.

Fig. 2. (a) Invariant cross sections as functions of $p_{T}$ at $\langle|y|\rangle=0.25$. The figure shows the cross sections for both charges at the three energies. The cross sections are scaled by factors of 10 for clarity. Different symbols denote the three energies and open and filled symbols distinguish between the two charges.

(b) Invariant cross sections as functions of $p_{T}$ at $\langle|y|\rangle=2.25$. The figure shows the cross sections for both charges and the three energies in the same way as in figure 2a.

Fig. 3. (a) Invariant cross sections as functions of $|y|$ at $\sqrt{s}=62 \mathrm{GeV}$ for positively charged particles. The figure shows the cross sections for the whole longitudinal phase space in eleven $p_{T}$-bins. Different symbols distinguish the different transverse momenta.

(b) Same as in Fig. 3(a), but for negatively charged particles.

Fig. 4. Charge separated invariant cross sections at $\mathrm{y}=0.25$ as functions of $\sqrt{s}$ for different $p_{T}$ values. Open (negative) and filled (positive) symbols are used to distinguish between the two charges.

Fig. 5. Invariant cross sections as functions of $y / y_{\max }$ for different $p_{T}$ regions normalized to 1 for $y / y_{\max }=0$. The values for the different values of $\sqrt{s}$ are shifted by 1 .

Fig. 6. (a) Invariant cross sections for positively charged particles at $\sqrt{s}=62 \mathrm{GeV}$ as functions of $p_{T}$ for different $y$ regions. The cross sections are scaled by a factor of 4 for clarity. Also shown is the result of the parametrization (full line) as described in section 4.3.

(b) Same as in Fig. 6(a), but for $\sqrt{s}=44 \mathrm{GeV}$.

(c) Same as in Fig. 6(a), but for $\sqrt{s}=31 \mathrm{GeV}$.

(d) Same as in Fig. 6(a), but for negatively charged particles.

(e) Same as in Fig. 6(d), but for $\sqrt{s}=44 \mathrm{GeV}$.

(f) Same as in Fig. 6(d), but for $\sqrt{s}=31 \mathrm{GeV}$. 


\begin{tabular}{|c|c|c|c|}
\hline$p_{T}$ & $\mathrm{y}$ & $E \cdot d^{3} \sigma / d p^{3} \oplus$ & $E \cdot d^{3} \sigma / d p^{3} \ominus$ \\
\hline$[\mathrm{GeV} / \mathrm{c}]$ & & {$\left[\mathrm{cm}^{2} \cdot \mathrm{c}^{3} / \mathrm{GeV}^{2}\right]$} & {$\left[\mathrm{cm}^{2} \cdot \mathrm{c}^{3} / \mathrm{GeV}^{2}\right]$} \\
\hline \multirow[t]{9}{*}{0.25} & 0.25 & $(4.986 \pm 0.051) 10^{-26}$ & $(4.848 \pm 0.049) 10^{-26}$ \\
\hline & 0.75 & $(4.574 \pm 0.049) 10^{-26}$ & $(4.692 \pm 0.075) 10^{-26}$ \\
\hline & 1.25 & $(3.701 \pm 0.037) 10^{-26}$ & $(3.943 \pm 0.038) 10^{-26}$ \\
\hline & 1.75 & $(3.572 \pm 0.024) 10^{-26}$ & $(3.103 \pm 0.028) 10^{-26}$ \\
\hline & 2.25 & $(3.040 \pm 0.024) 10^{-26}$ & $(2.654 \pm 0.017) 10^{-26}$ \\
\hline & 2.75 & $(2.429 \pm 0.018) 10^{-26}$ & $(2.220 \pm 0.014) 10^{-26}$ \\
\hline & 3.25 & $(1.978 \pm 0.013) 10^{-26}$ & $(8.588 \pm 0.069) 10^{-27}$ \\
\hline & 3.75 & $(1.479 \pm 0.010) 10^{-26}$ & $(4.572 \pm 0.046) 10^{-27}$ \\
\hline & 4.25 & $(9.983 \pm 0.085) 10^{-27}$ & $(1.373 \pm 0.025) 10^{-27}$ \\
\hline \multirow[t]{9}{*}{0.35} & 0.25 & $(2.611 \pm 0.030) 10^{-26}$ & $(2.722 \pm 0.034) 10^{-26}$ \\
\hline & 0.75 & $(2.719 \pm 0.033) 10^{-26}$ & $(2.358 \pm 0.032) 10^{-26}$ \\
\hline & 1.25 & $(2.141 \pm 0.021) 10^{-26}$ & $(2.248 \pm 0.022) 10^{-26}$ \\
\hline & 1.75 & $(2.066 \pm 0.014) 10^{-26}$ & $(1.712 \pm 0.014) 10^{-26}$ \\
\hline & 2.25 & $(1.864 \pm 0.014) 10^{-26}$ & $(1.625 \pm 0.011) 10^{-26}$ \\
\hline & 2.75 & $(1.407 \pm 0.010) 10^{-26}$ & $(1.160 \pm 0.008) 10^{-26}$ \\
\hline & 3.25 & $(1.183 \pm 0.008) 10^{-26}$ & $(5.496 \pm 0.049) 10^{-27}$ \\
\hline & 3.75 & $(8.918 \pm 0.071) 10^{-27}$ & $(2.033 \pm 0.027) 10^{-27}$ \\
\hline & 4.25 & $(6.563 \pm 0.063) 10^{-27}$ & $(0.554 \pm 0.014) 10^{-27}$ \\
\hline \multirow[t]{9}{*}{0.45} & 0.25 & $(1.427 \pm 0.019) 10^{-26}$ & $(1.414 \pm 0.023) 10^{-26}$ \\
\hline & 0.75 & $(1.520 \pm 0.021) 10^{-26}$ & $(1.388 \pm 0.022) 10^{-26}$ \\
\hline & 1.25 & $(1.201 \pm 0.014) 10^{-26}$ & $(1.240 \pm 0.016) 10^{-26}$ \\
\hline & 1.75 & $(1.186 \pm 0.010) 10^{-26}$ & $(9.826 \pm 0.097) 10^{-27}$ \\
\hline & 2.25 & $(1.000 \pm 0.008) 10^{-26}$ & $(8.809 \pm 0.073) 10^{-27}$ \\
\hline & 2.75 & $(8.405 \pm 0.066) 10^{-27}$ & $(6.415 \pm 0.051) 10^{-27}$ \\
\hline & 3.25 & $(6.696 \pm 0.056) 10^{-27}$ & $(2.941 \pm 0.033) 10^{-27}$ \\
\hline & 3.75 & $(5.403 \pm 0.049) 10^{-27}$ & $(0.830 \pm 0.015) 10^{-27}$ \\
\hline & 4.25 & $(4.222 \pm 0.042) 10^{-27}$ & $(2.128 \pm 0.070) 10^{-28}$ \\
\hline \multirow[t]{9}{*}{0.55} & 0.25 & $(0.823 \pm 0.011) 10^{-26}$ & $(0.773 \pm 0.012) 10^{-26}$ \\
\hline & 0.75 & $(0.896 \pm 0.013) 10^{-26}$ & $(0.741 \pm 0.012) 10^{-26}$ \\
\hline & 1.25 & $(0.769 \pm 0.011) 10^{-26}$ & $(0.742 \pm 0.011) 10^{-26}$ \\
\hline & 1.75 & $(6.769 \pm 0.058) 10^{-27}$ & $(5.482 \pm 0.053) 10^{-27}$ \\
\hline & 2.25 & $(5.832 \pm 0.046) 10^{-27}$ & $(4.933 \pm 0.043) 10^{-27}$ \\
\hline & 2.75 & $(5.224 \pm 0.043) 10^{-27}$ & $(3.569 \pm 0.032) 10^{-27}$ \\
\hline & 3.25 & $(4.407 \pm 0.037) 10^{-27}$ & $(1.571 \pm 0.019) 10^{-27}$ \\
\hline & 3.75 & $(3.663 \pm 0.035) 10^{-27}$ & $(4.635 \pm 0.099) 10^{-28}$ \\
\hline & 4.25 & $(2.511 \pm 0.028) 10^{-27}$ & $(0.631 \pm 0.033) 10^{-28}$ \\
\hline \multirow[t]{9}{*}{0.65} & 0.25 & $(4.732 \pm 0.066) 10^{-27}$ & $(4.424 \pm 0.068) 10^{-27}$ \\
\hline & 0.75 & $(5.256 \pm 0.079) 10^{-27}$ & $(4.166 \pm 0.073) 10^{-27}$ \\
\hline & 1.25 & $(4.355 \pm 0.063) 10^{-27}$ & $(3.833 \pm 0.051) 10^{-27}$ \\
\hline & 1.75 & $(4.175 \pm 0.040) 10^{-27}$ & $(3.119 \pm 0.034) 10^{-27}$ \\
\hline & 2.25 & $(3.637 \pm 0.032) 10^{-27}$ & $(2.695 \pm 0.027) 10^{-27}$ \\
\hline & 2.75 & $(3.205 \pm 0.026) 10^{-27}$ & $(1.874 \pm 0.020) 10^{-27}$ \\
\hline & 3.25 & $(2.822 \pm 0.027) 10^{-27}$ & $(0.824 \pm 0.013) 10^{-27}$ \\
\hline & 3.75 & $(2.192 \pm 0.023) 10^{-27}$ & $(1.938 \pm 0.055) 10^{-28}$ \\
\hline & 4.25 & $(1.468 \pm 0.019) 10^{-27}$ & $(0.200 \pm 0.018) 10^{-28}$ \\
\hline
\end{tabular}




\begin{tabular}{|c|c|c|c|}
\hline$p_{T}$ & $\mathrm{y}$ & $E \cdot d^{3} \sigma / d p^{3} \oplus$ & $E \cdot d^{3} \sigma / d p^{3} \ominus$ \\
\hline$[\mathrm{GeV} / \mathrm{c}]$ & & {$\left[\mathrm{cm}^{2} \cdot \mathrm{c}^{3} / \mathrm{GeV}^{2}\right]$} & {$\left[\mathrm{cm}^{2} \cdot \mathrm{c}^{3} / \mathrm{GeV}^{2}\right]$} \\
\hline \multirow[t]{8}{*}{0.82} & 0.25 & $(1.904 \pm 0.034) 10^{-27}$ & $(1.667 \pm 0.036) 10^{-27}$ \\
\hline & 0.75 & $(1.944 \pm 0.031) 10^{-27}$ & $(1.681 \pm 0.036) 10^{-27}$ \\
\hline & 1.25 & $(1.751 \pm 0.028) 10^{-27}$ & $(1.468 \pm 0.025) 10^{-27}$ \\
\hline & 1.75 & $(1.618 \pm 0.017) 10^{-27}$ & $(1.186 \pm 0.015) 10^{-27}$ \\
\hline & 2.25 & $(1.493 \pm 0.013) 10^{-27}$ & $(9.461 \pm 0.099) 10^{-28}$ \\
\hline & 2.75 & $(1.278 \pm 0.010) 10^{-27}$ & $(6.103 \pm 0.066) 10^{-28}$ \\
\hline & 3.25 & $(1.121 \pm 0.011) 10^{-27}$ & $(2.184 \pm 0.036) 10^{-28}$ \\
\hline & 3.75 & $(8.808 \pm 0.095) 10^{-28}$ & $(0.449 \pm 0.015) 10^{-28}$ \\
\hline \multirow[t]{8}{*}{1.12} & 0.25 & $(4.520 \pm 0.098) 10^{-28}$ & $(0.368 \pm 0.010) 10^{-27}$ \\
\hline & 0.75 & $(4.885 \pm 0.095) 10^{-28}$ & $(3.771 \pm 0.094) 10^{-28}$ \\
\hline & 1.25 & $(4.421 \pm 0.082) 10^{-28}$ & $(3.312 \pm 0.071) 10^{-28}$ \\
\hline & 1.75 & $(3.664 \pm 0.050) 10^{-28}$ & $(2.443 \pm 0.040) 10^{-28}$ \\
\hline & 2.25 & $(3.201 \pm 0.044) 10^{-28}$ & $(1.634 \pm 0.028) 10^{-28}$ \\
\hline & 2.75 & $(2.457 \pm 0.031) 10^{-28}$ & $(0.974 \pm 0.019) 10^{-28}$ \\
\hline & 3.25 & $(2.110 \pm 0.029) 10^{-28}$ & $(0.317 \pm 0.011) 10^{-28}$ \\
\hline & 3.75 & $(1.280 \pm 0.025) 10^{-28}$ & $(0.451 \pm 0.040) 10^{-29}$ \\
\hline \multirow[t]{7}{*}{1.42} & 0.25 & $(1.255 \pm 0.041) 10^{-28}$ & $(1.113 \pm 0.043) 10^{-28}$ \\
\hline & 0.75 & $(1.289 \pm 0.035) 10^{-28}$ & $(1.040 \pm 0.036) 10^{-28}$ \\
\hline & 1.25 & $(1.162 \pm 0.028) 10^{-28}$ & $(0.869 \pm 0.025) 10^{-28}$ \\
\hline & 1.75 & $(0.976 \pm 0.019) 10^{-28}$ & $(0.563 \pm 0.014) 10^{-28}$ \\
\hline & 2.25 & $(0.690 \pm 0.015) 10^{-28}$ & $(3.349 \pm 0.093) 10^{-29}$ \\
\hline & 2.75 & $(0.466 \pm 0.011) 10^{-28}$ & $(1.765 \pm 0.064) 10^{-29}$ \\
\hline & 3.25 & $(3.428 \pm 0.093) 10^{-29}$ & $(0.876 \pm 0.047) 10^{-29}$ \\
\hline \multirow[t]{7}{*}{1.72} & 0.25 & $(0.396 \pm 0.024) 10^{-28}$ & $(0.291 \pm 0.018) 10^{-28}$ \\
\hline & 0.75 & $(0.380 \pm 0.016) 10^{-28}$ & $(0.307 \pm 0.016) 10^{-28}$ \\
\hline & 1.25 & $(0.354 \pm 0.012) 10^{-28}$ & $(2.471 \pm 0.099) 10^{-29}$ \\
\hline & 1.75 & $(2.528 \pm 0.073) 10^{-29}$ & $(1.452 \pm 0.055) 10^{-29}$ \\
\hline & 2.25 & $(1.796 \pm 0.062) 10^{-29}$ & $(0.760 \pm 0.039) 10^{-29}$ \\
\hline & 2.75 & $(0.969 \pm 0.043) 10^{-29}$ & $(0.425 \pm 0.028) 10^{-29}$ \\
\hline & 3.25 & $(0.595 \pm 0.032) 10^{-29}$ & $(0.318 \pm 0.026) 10^{-29}$ \\
\hline \multirow[t]{6}{*}{2.18} & 0.25 & $(0.550 \pm 0.049) 10^{-29}$ & $(0.475 \pm 0.051) 10^{-29}$ \\
\hline & 0.75 & $(0.639 \pm 0.050) 10^{-29}$ & $(0.509 \pm 0.035) 10^{-29}$ \\
\hline & 1.25 & $(0.663 \pm 0.036) 10^{-29}$ & $(0.402 \pm 0.023) 10^{-29}$ \\
\hline & 1.75 & $(0.376 \pm 0.019) 10^{-29}$ & $(0.209 \pm 0.013) 10^{-29}$ \\
\hline & 2.25 & $(0.261 \pm 0.014) 10^{-29}$ & $(0.996 \pm 0.074) 10^{-30}$ \\
\hline & 2.75 & $(1.283 \pm 0.084) 10^{-30}$ & $(0.722 \pm 0.063) 10^{-30}$ \\
\hline \multirow[t]{6}{*}{3.00} & 0.25 & $(0.029 \pm 0.010) 10^{-29}$ & $(0.268 \pm 0.079) 10^{-30}$ \\
\hline & 0.75 & $(0.453 \pm 0.086) 10^{-30}$ & $(0.482 \pm 0.064) 10^{-30}$ \\
\hline & 1.25 & $(0.578 \pm 0.120) 10^{-30}$ & $(0.325 \pm 0.077) 10^{-30}$ \\
\hline & 1.75 & $(0.194 \pm 0.045) 10^{-30}$ & $(0.117 \pm 0.031) 10^{-30}$ \\
\hline & 2.25 & $(0.151 \pm 0.025) 10^{-30}$ & $(0.083 \pm 0.016) 10^{-30}$ \\
\hline & 2.75 & $(0.079 \pm 0.016) 10^{-30}$ & $(0.097 \pm 0.019) 10^{-30}$ \\
\hline
\end{tabular}

Table 1: 


\begin{tabular}{|c|c|c|c|}
\hline$p_{T}$ & $\mathrm{y}$ & $E \cdot d^{3} \sigma / d p^{3} \oplus$ & $E \cdot d^{3} \sigma / d p^{3} \ominus$ \\
\hline$[\mathrm{GeV} / \mathrm{c}]$ & & {$\left[\mathrm{cm}^{2} \cdot \mathrm{c}^{3} / \mathrm{GeV}^{2}\right]$} & {$\left[\mathrm{cm}^{2} \cdot \mathrm{c}^{3} / \mathrm{GeV}^{2}\right]$} \\
\hline \multirow[t]{9}{*}{0.25} & 0.25 & $(4.337 \pm 0.050) 10^{-26}$ & $(4.327 \pm 0.053) 10^{-26}$ \\
\hline & 0.75 & $(4.178 \pm 0.053) 10^{-26}$ & $(4.424 \pm 0.082) 10^{-26}$ \\
\hline & 1.25 & $(3.435 \pm 0.033) 10^{-26}$ & $(3.754 \pm 0.036) 10^{-26}$ \\
\hline & 1.75 & $(3.388 \pm 0.024) 10^{-26}$ & $(2.783 \pm 0.024) 10^{-26}$ \\
\hline & 2.25 & $(2.920 \pm 0.022) 10^{-26}$ & $(2.287 \pm 0.015) 10^{-26}$ \\
\hline & 2.75 & $(2.063 \pm 0.017) 10^{-26}$ & $(1.661 \pm 0.013) 10^{-26}$ \\
\hline & 3.25 & $(1.612 \pm 0.012) 10^{-26}$ & $(6.600 \pm 0.068) 10^{-27}$ \\
\hline & 3.75 & $(1.180 \pm 0.011) 10^{-26}$ & $(2.786 \pm 0.040) 10^{-27}$ \\
\hline & 4.25 & $(8.298 \pm 0.085) 10^{-27}$ & $(0.660 \pm 0.019) 10^{-27}$ \\
\hline \multirow[t]{9}{*}{0.35} & 0.25 & $(2.266 \pm 0.025) 10^{-26}$ & $(2.317 \pm 0.029) 10^{-26}$ \\
\hline & 0.75 & $(2.414 \pm 0.028) 10^{-26}$ & $(2.192 \pm 0.029) 10^{-26}$ \\
\hline & 1.25 & $(2.004 \pm 0.022) 10^{-26}$ & $(2.025 \pm 0.019) 10^{-26}$ \\
\hline & 1.75 & $(1.827 \pm 0.012) 10^{-26}$ & $(1.503 \pm 0.012) 10^{-26}$ \\
\hline & 2.25 & $(1.597 \pm 0.012) 10^{-26}$ & $(1.279 \pm 0.009) 10^{-26}$ \\
\hline & 2.75 & $(1.204 \pm 0.009) 10^{-26}$ & $(8.330 \pm 0.066) 10^{-27}$ \\
\hline & 3.25 & $(9.379 \pm 0.079) 10^{-27}$ & $(3.507 \pm 0.041) 10^{-27}$ \\
\hline & 3.75 & $(7.350 \pm 0.067) 10^{-27}$ & $(0.947 \pm 0.020) 10^{-27}$ \\
\hline & 4.25 & $(6.293 \pm 0.065) 10^{-27}$ & $(1.826 \pm 0.088) 10^{-28}$ \\
\hline \multirow[t]{9}{*}{0.45} & 0.25 & $(1.300 \pm 0.015) 10^{-26}$ & $(1.232 \pm 0.016) 10^{-26}$ \\
\hline & 0.75 & $(1.389 \pm 0.016) 10^{-26}$ & $(1.220 \pm 0.017) 10^{-26}$ \\
\hline & 1.25 & $(1.152 \pm 0.013) 10^{-26}$ & $(1.139 \pm 0.012) 10^{-26}$ \\
\hline & 1.75 & $(1.044 \pm 0.008) 10^{-26}$ & $(8.470 \pm 0.073) 10^{-27}$ \\
\hline & 2.25 & $(9.367 \pm 0.070) 10^{-27}$ & $(7.304 \pm 0.056) 10^{-27}$ \\
\hline & 2.75 & $(7.352 \pm 0.055) 10^{-27}$ & $(4.433 \pm 0.039) 10^{-27}$ \\
\hline & 3.25 & $(5.745 \pm 0.047) 10^{-27}$ & $(1.744 \pm 0.024) 10^{-27}$ \\
\hline & 3.75 & $(4.692 \pm 0.043) 10^{-27}$ & $(0.390 \pm 0.011) 10^{-27}$ \\
\hline & 4.25 & $(4.233 \pm 0.043) 10^{-27}$ & $(0.384 \pm 0.033) 10^{-28}$ \\
\hline \multirow[t]{9}{*}{0.55} & 0.25 & $(7.110 \pm 0.091) 10^{-27}$ & $(0.676 \pm 0.010) 10^{-26}$ \\
\hline & 0.75 & $(7.835 \pm 0.096) 10^{-27}$ & $(6.332 \pm 0.090) 10^{-27}$ \\
\hline & 1.25 & $(6.715 \pm 0.083) 10^{-27}$ & $(6.149 \pm 0.072) 10^{-27}$ \\
\hline & 1.75 & $(6.023 \pm 0.050) 10^{-27}$ & $(4.867 \pm 0.046) 10^{-27}$ \\
\hline & 2.25 & $(5.517 \pm 0.045) 10^{-27}$ & $(3.983 \pm 0.036) 10^{-27}$ \\
\hline & 2.75 & $(4.922 \pm 0.038) 10^{-27}$ & $(2.398 \pm 0.025) 10^{-27}$ \\
\hline & 3.25 & $(3.933 \pm 0.035) 10^{-27}$ & $(0.883 \pm 0.015) 10^{-27}$ \\
\hline & 3.75 & $(3.055 \pm 0.031) 10^{-27}$ & $(1.468 \pm 0.059) 10^{-28}$ \\
\hline & 4.25 & $(2.660 \pm 0.032) 10^{-27}$ & $(0.080 \pm 0.013) 10^{-28}$ \\
\hline \multirow[t]{8}{*}{0.65} & 0.25 & $(4.372 \pm 0.067) 10^{-27}$ & $(3.908 \pm 0.067) 10^{-27}$ \\
\hline & 0.75 & $(4.675 \pm 0.063) 10^{-27}$ & $(3.699 \pm 0.062) 10^{-27}$ \\
\hline & 1.25 & $(3.649 \pm 0.046) 10^{-27}$ & $(3.363 \pm 0.042) 10^{-27}$ \\
\hline & 1.75 & $(3.503 \pm 0.034) 10^{-27}$ & $(2.635 \pm 0.029) 10^{-27}$ \\
\hline & 2.25 & $(3.351 \pm 0.031) 10^{-27}$ & $(1.962 \pm 0.022) 10^{-27}$ \\
\hline & 2.75 & $(3.079 \pm 0.026) 10^{-27}$ & $(1.247 \pm 0.016) 10^{-27}$ \\
\hline & 3.25 & $(2.656 \pm 0.026) 10^{-27}$ & $(3.849 \pm 0.092) 10^{-28}$ \\
\hline & 3.75 & $(1.847 \pm 0.021) 10^{-27}$ & $(0.473 \pm 0.030) 10^{-28}$ \\
\hline
\end{tabular}




\begin{tabular}{|c|c|c|c|}
\hline$p_{T}$ & $\mathrm{y}$ & $E \cdot d^{3} \sigma / d p^{3} \oplus$ & $E \cdot d^{3} \sigma / d p^{3} \ominus$ \\
\hline$[\mathrm{GeV} / \mathrm{c}]$ & & {$\left[\mathrm{cm}^{2} \cdot \mathrm{c}^{3} / \mathrm{GeV}^{2}\right]$} & {$\left[\mathrm{cm}^{2} \cdot \mathrm{c}^{3} / \mathrm{GeV}^{2}\right]$} \\
\hline \multirow[t]{8}{*}{0.82} & 0.25 & $(1.729 \pm 0.031) 10^{-27}$ & $(1.468 \pm 0.029) 10^{-27}$ \\
\hline & 0.75 & $(1.784 \pm 0.029) 10^{-27}$ & $(1.357 \pm 0.026) 10^{-27}$ \\
\hline & 1.25 & $(1.522 \pm 0.024) 10^{-27}$ & $(1.240 \pm 0.019) 10^{-27}$ \\
\hline & 1.75 & $(1.393 \pm 0.015) 10^{-27}$ & $(0.919 \pm 0.010) 10^{-27}$ \\
\hline & 2.25 & $(1.290 \pm 0.013) 10^{-27}$ & $(6.647 \pm 0.075) 10^{-28}$ \\
\hline & 2.75 & $(1.178 \pm 0.010) 10^{-27}$ & $(3.469 \pm 0.046) 10^{-28}$ \\
\hline & 3.25 & $(1.048 \pm 0.010) 10^{-27}$ & $(0.994 \pm 0.025) 10^{-28}$ \\
\hline & 3.75 & $(5.960 \pm 0.077) 10^{-28}$ & $(0.874 \pm 0.074) 10^{-} 29$ \\
\hline \multirow[t]{8}{*}{1.12} & 0.25 & $(3.941 \pm 0.103) 10^{-28}$ & $(3.210 \pm 0.089) 10^{-28}$ \\
\hline & 0.75 & $(3.817 \pm 0.086) 10^{-28}$ & $(3.022 \pm 0.078) 10^{-28}$ \\
\hline & 1.25 & $(3.658 \pm 0.073) 10^{-28}$ & $(2.527 \pm 0.053) 10^{-28}$ \\
\hline & 1.75 & $(3.116 \pm 0.046) 10^{-28}$ & $(1.755 \pm 0.033) 10^{-28}$ \\
\hline & 2.25 & $(2.432 \pm 0.036) 10^{-28}$ & $(0.991 \pm 0.021) 10^{-28}$ \\
\hline & 2.75 & $(2.152 \pm 0.032) 10^{-28}$ & $(0.454 \pm 0.013) 10^{-28}$ \\
\hline & 3.25 & $(1.589 \pm 0.027) 10^{-28}$ & $(1.011 \pm 0.063) 10^{-29}$ \\
\hline & 3.75 & $(0.481 \pm 0.073) 10^{-28}$ & $(0.078 \pm 0.023) 10^{-29}$ \\
\hline \multirow[t]{7}{*}{1.42} & 0.25 & $(1.053 \pm 0.047) 10^{-28}$ & $(0.924 \pm 0.039) 10^{-28}$ \\
\hline & 0.75 & $(1.015 \pm 0.036) 10^{-28}$ & $(0.746 \pm 0.029) 10^{-28}$ \\
\hline & 1.25 & $(0.900 \pm 0.025) 10^{-28}$ & $(0.641 \pm 0.022) 10^{-28}$ \\
\hline & 1.75 & $(0.691 \pm 0.016) 10^{-28}$ & $(0.383 \pm 0.012) 10^{-28}$ \\
\hline & 2.25 & $(0.522 \pm 0.013) 10^{-28}$ & $(1.742 \pm 0.073) 10^{-29}$ \\
\hline & 2.75 & $(0.363 \pm 0.010) 10^{-28}$ & $(0.671 \pm 0.045) 10^{-29}$ \\
\hline & 3.25 & $(1.817 \pm 0.073) 10^{-29}$ & $(0.225 \pm 0.026) 10^{-29}$ \\
\hline \multirow[t]{7}{*}{1.72} & 0.25 & $(0.264 \pm 0.018) 10^{-28}$ & $(0.214 \pm 0.014) 10^{-28}$ \\
\hline & 0.75 & $(0.261 \pm 0.014) 10^{-28}$ & $(0.203 \pm 0.013) 10^{-28}$ \\
\hline & 1.25 & $(0.288 \pm 0.013) 10^{-28}$ & $(1.517 \pm 0.085) 10^{-29}$ \\
\hline & 1.75 & $(1.584 \pm 0.062) 10^{-29}$ & $(0.841 \pm 0.045) 10^{-29}$ \\
\hline & 2.25 & $(1.327 \pm 0.057) 10^{-29}$ & $(0.366 \pm 0.029) 10^{-29}$ \\
\hline & 2.75 & $(0.603 \pm 0.037) 10^{-29}$ & $(0.116 \pm 0.017) 10^{-29}$ \\
\hline & 3.25 & $(0.223 \pm 0.022) 10^{-29}$ & $(0.133 \pm 0.018) 10^{-29}$ \\
\hline \multirow[t]{7}{*}{2.18} & 0.25 & $(0.390 \pm 0.033) 10^{-29}$ & $(0.335 \pm 0.033) 10^{-29}$ \\
\hline & 0.75 & $(0.478 \pm 0.035) 10^{-29}$ & $(0.345 \pm 0.027) 10^{-29}$ \\
\hline & 1.25 & $(0.443 \pm 0.025) 10^{-29}$ & $(0.289 \pm 0.020) 10^{-29}$ \\
\hline & 1.75 & $(0.223 \pm 0.015) 10^{-29}$ & $(0.119 \pm 0.010) 10^{-29}$ \\
\hline & 2.25 & $(1.497 \pm 0.098) 10^{-30}$ & $(0.412 \pm 0.048) 10^{-30}$ \\
\hline & 2.75 & $(0.544 \pm 0.061) 10^{-30}$ & $(0.174 \pm 0.034) 10^{-30}$ \\
\hline & 3.25 & $(0.092 \pm 0.019) 10^{-30}$ & $(0.117 \pm 0.030) 10^{-30}$ \\
\hline \multirow[t]{5}{*}{3.00} & 0.25 & $(0.249 \pm 0.076) 10^{-30}$ & $(0.241 \pm 0.111) 10^{-30}$ \\
\hline & 0.75 & $(0.247 \pm 0.051) 10^{-30}$ & $(0.191 \pm 0.041) 10^{-30}$ \\
\hline & 1.25 & $(0.332 \pm 0.064) 10^{-30}$ & $(0.205 \pm 0.210) 10^{-30}$ \\
\hline & 1.75 & $(0.145 \pm 0.025) 10^{-30}$ & $(0.053 \pm 0.014) 10^{-30}$ \\
\hline & 2.25 & $(0.060 \pm 0.012) 10^{-30}$ & $(0.252 \pm 0.077) 10^{-31}$ \\
\hline
\end{tabular}

Table 2: 


\begin{tabular}{|c|c|c|c|}
\hline$p_{T}$ & $\mathrm{y}$ & $E \cdot d^{3} \sigma / d p^{3} \oplus$ & $E \cdot d^{3} \sigma / d p^{3} \ominus$ \\
\hline$[\mathrm{GeV} / \mathrm{c}]$ & & {$\left[\mathrm{cm}^{2} \cdot \mathrm{c}^{3} / \mathrm{GeV}^{2}\right]$} & {$\left[\mathrm{cm}^{2} \cdot \mathrm{c}^{3} / \mathrm{GeV}^{2}\right]$} \\
\hline \multirow[t]{8}{*}{0.25} & 0.25 & $(4.030 \pm 0.046) 10^{-26}$ & $\overline{(3.822 \pm 0.053) 10^{-26}}$ \\
\hline & 0.75 & $(4.261 \pm 0.048) 10^{-26}$ & $(3.765 \pm 0.050) 10^{-26}$ \\
\hline & 1.25 & $(3.201 \pm 0.025) 10^{-26}$ & $(3.307 \pm 0.027) 10^{-26}$ \\
\hline & 1.75 & $(3.066 \pm 0.016) 10^{-26}$ & $(2.515 \pm 0.017) 10^{-26}$ \\
\hline & 2.25 & $(2.331 \pm 0.012) 10^{-26}$ & $(1.881 \pm 0.010) 10^{-26}$ \\
\hline & 2.75 & $(1.745 \pm 0.010) 10^{-26}$ & $(1.129 \pm 0.008) 10^{-26}$ \\
\hline & 3.25 & $(1.287 \pm 0.008) 10^{-26}$ & $(3.541 \pm 0.035) 10^{-27}$ \\
\hline & 3.75 & $(9.855 \pm 0.077) 10^{-27}$ & $(1.100 \pm 0.017) 10^{-27}$ \\
\hline \multirow[t]{8}{*}{0.35} & 0.25 & $(2.076 \pm 0.023) 10^{-26}$ & $(1.949 \pm 0.024) 10^{-26}$ \\
\hline & 0.75 & $(2.358 \pm 0.024) 10^{-26}$ & $(1.951 \pm 0.022) 10^{-26}$ \\
\hline & 1.25 & $(1.887 \pm 0.016) 10^{-26}$ & $(1.682 \pm 0.014) 10^{-26}$ \\
\hline & 1.75 & $(1.698 \pm 0.010) 10^{-26}$ & $(1.269 \pm 0.008) 10^{-26}$ \\
\hline & 2.25 & $(1.404 \pm 0.008) 10^{-26}$ & $(1.005 \pm 0.006) 10^{-26}$ \\
\hline & 2.75 & $(1.119 \pm 0.006) 10^{-26}$ & $(5.513 \pm 0.037) 10^{-27}$ \\
\hline & 3.25 & $(8.229 \pm 0.049) 10^{-27}$ & $(1.722 \pm 0.018) 10^{-27}$ \\
\hline & 3.75 & $(6.770 \pm 0.047) 10^{-27}$ & $(3.353 \pm 0.075) 10^{-28}$ \\
\hline \multirow[t]{8}{*}{0.45} & 0.25 & $(1.331 \pm 0.017) 10^{-26}$ & $(1.152 \pm 0.017) 10^{-26}$ \\
\hline & 0.75 & $(1.309 \pm 0.015) 10^{-26}$ & $(1.133 \pm 0.014) 10^{-26}$ \\
\hline & 1.25 & $(1.071 \pm 0.010) 10^{-26}$ & $(1.005 \pm 0.010) 10^{-26}$ \\
\hline & 1.75 & $(9.941 \pm 0.062) 10^{-27}$ & $(7.281 \pm 0.050) 10^{-27}$ \\
\hline & 2.25 & $(8.817 \pm 0.049) 10^{-27}$ & $(5.467 \pm 0.034) 10^{-27}$ \\
\hline & 2.75 & $(7.003 \pm 0.038) 10^{-27}$ & $(2.538 \pm 0.019) 10^{-27}$ \\
\hline & 3.25 & $(5.682 \pm 0.036) 10^{-27}$ & $(0.766 \pm 0.010) 10^{-27}$ \\
\hline & 3.75 & $(4.637 \pm 0.034) 10^{-27}$ & $(1.065 \pm 0.039) 10^{-28}$ \\
\hline \multirow[t]{8}{*}{0.55} & 0.25 & $(6.451 \pm 0.076) 10^{-27}$ & $(6.660 \pm 0.096) 10^{-27}$ \\
\hline & 0.75 & $(7.782 \pm 0.091) 10^{-27}$ & $(5.855 \pm 0.080) 10^{-27}$ \\
\hline & 1.25 & $(6.682 \pm 0.070) 10^{-27}$ & $(5.408 \pm 0.059) 10^{-27}$ \\
\hline & 1.75 & $(5.921 \pm 0.042) 10^{-27}$ & $(4.014 \pm 0.030) 10^{-27}$ \\
\hline & 2.25 & $(5.293 \pm 0.032) 10^{-27}$ & $(2.821 \pm 0.021) 10^{-27}$ \\
\hline & 2.75 & $(4.397 \pm 0.024) 10^{-27}$ & $(1.227 \pm 0.011) 10^{-27}$ \\
\hline & 3.25 & $(3.831 \pm 0.025) 10^{-27}$ & $(3.113 \pm 0.058) 10^{-28}$ \\
\hline & 3.75 & $(2.828 \pm 0.022) 10^{-27}$ & $(0.272 \pm 0.018) 10^{-28}$ \\
\hline \multirow[t]{8}{*}{0.65} & 0.25 & $(4.251 \pm 0.076) 10^{-27}$ & $(3.918 \pm 0.063) 10^{-27}$ \\
\hline & 0.75 & $(4.396 \pm 0.054) 10^{-27}$ & $(3.297 \pm 0.049) 10^{-27}$ \\
\hline & 1.25 & $(3.809 \pm 0.041) 10^{-27}$ & $(3.031 \pm 0.033) 10^{-27}$ \\
\hline & 1.75 & $(3.465 \pm 0.027) 10^{-27}$ & $(2.140 \pm 0.019) 10^{-27}$ \\
\hline & 2.25 & $(2.848 \pm 0.020) 10^{-27}$ & $(1.389 \pm 0.012) 10^{-27}$ \\
\hline & 2.75 & $(2.835 \pm 0.018) 10^{-27}$ & $(6.186 \pm 0.072) 10^{-28}$ \\
\hline & 3.25 & $(2.317 \pm 0.016) 10^{-27}$ & $(1.222 \pm 0.032) 10^{-28}$ \\
\hline & 3.75 & $(1.388 \pm 0.013) 10^{-27}$ & $(0.648 \pm 0.074) 10^{-29}$ \\
\hline
\end{tabular}




\begin{tabular}{|c|c|c|c|}
\hline$p_{T}$ & $\mathrm{y}$ & $E \cdot d^{3} \sigma / d p^{3} \oplus$ & $E \cdot d^{3} \sigma / d p^{3} \ominus$ \\
\hline$[\mathrm{GeV} / \mathrm{c}]$ & & {$\left[\mathrm{cm}^{2} \cdot \mathrm{c}^{3} / \mathrm{GeV}^{2}\right]$} & {$\left[\mathrm{cm}^{2} \cdot \mathrm{c}^{3} / \mathrm{GeV}^{2}\right]$} \\
\hline \multirow[t]{7}{*}{0.82} & 0.25 & $(1.574 \pm 0.034) 10^{-27}$ & $(1.317 \pm 0.029) 10^{-27}$ \\
\hline & 0.75 & $(1.524 \pm 0.021) 10^{-27}$ & $(1.225 \pm 0.022) 10^{-27}$ \\
\hline & 1.25 & $(1.408 \pm 0.018) 10^{-27}$ & $(1.036 \pm 0.015) 10^{-27}$ \\
\hline & 1.75 & $(1.216 \pm 0.012) 10^{-27}$ & $(6.837 \pm 0.068) 10^{-28}$ \\
\hline & 2.25 & $(1.125 \pm 0.009) 10^{-27}$ & $(4.204 \pm 0.040) 10^{-28}$ \\
\hline & 2.75 & $(1.155 \pm 0.008) 10^{-27}$ & $(1.582 \pm 0.020) 10^{-28}$ \\
\hline & 3.25 & $(7.973 \pm 0.066) 10^{-28}$ & $(2.313 \pm 0.074) 10^{-29}$ \\
\hline \multirow[t]{6}{*}{1.12} & 0.25 & $(0.365 \pm 0.010) 10^{-27}$ & $(2.528 \pm 0.053) 10^{-28}$ \\
\hline & 0.75 & $(3.369 \pm 0.070) 10^{-28}$ & $(2.568 \pm 0.062) 10^{-28}$ \\
\hline & 1.25 & $(3.370 \pm 0.062) 10^{-28}$ & $(2.094 \pm 0.042) 10^{-28}$ \\
\hline & 1.75 & $(2.435 \pm 0.030) 10^{-28}$ & $(1.137 \pm 0.018) 10^{-28}$ \\
\hline & 2.25 & $(2.338 \pm 0.027) 10^{-28}$ & $(5.370 \pm 0.099) 10^{-29}$ \\
\hline & 2.75 & $(1.964 \pm 0.021) 10^{-28}$ & $(1.272 \pm 0.044) 10^{-29}$ \\
\hline \multirow[t]{6}{*}{1.42} & 0.25 & $(0.959 \pm 0.030) 10^{-28}$ & $(0.689 \pm 0.025) 10^{-28}$ \\
\hline & 0.75 & $(0.836 \pm 0.023) 10^{-28}$ & $(0.621 \pm 0.020) 10^{-28}$ \\
\hline & 1.25 & $(0.777 \pm 0.017) 10^{-28}$ & $(0.438 \pm 0.012) 10^{-28}$ \\
\hline & 1.75 & $(5.311 \pm 0.092) 10^{-29}$ & $(2.446 \pm 0.062) 10^{-29}$ \\
\hline & 2.25 & $(4.906 \pm 0.087) 10^{-29}$ & $(0.729 \pm 0.030) 10^{-29}$ \\
\hline & 2.75 & $(2.505 \pm 0.057) 10^{-29}$ & $(0.164 \pm 0.014) 10^{-29}$ \\
\hline \multirow[t]{6}{*}{1.72} & 0.25 & $(0.232 \pm 0.010) 10^{-28}$ & $(0.166 \pm 0.010) 10^{-28}$ \\
\hline & 0.75 & $(0.226 \pm 0.010) 10^{-28}$ & $(1.600 \pm 0.090) 10^{-29}$ \\
\hline & 1.25 & $(1.882 \pm 0.062) 10^{-29}$ & $(0.916 \pm 0.043) 10^{-29}$ \\
\hline & 1.75 & $(1.484 \pm 0.041) 10^{-29}$ & $(0.647 \pm 0.027) 10^{-29}$ \\
\hline & 2.25 & $(0.939 \pm 0.031) 10^{-29}$ & $(0.121 \pm 0.010) 10^{-29}$ \\
\hline & 2.75 & $(0.319 \pm 0.018) 10^{-29}$ & $(0.258 \pm 0.045) 10^{-30}$ \\
\hline \multirow[t]{5}{*}{2.18} & 0.25 & $(0.300 \pm 0.037) 10^{-29}$ & $(0.243 \pm 0.024) 10^{-29}$ \\
\hline & 0.75 & $(0.332 \pm 0.022) 10^{-29}$ & $(0.261 \pm 0.018) 10^{-29}$ \\
\hline & 1.25 & $(0.273 \pm 0.015) 10^{-29}$ & $(1.488 \pm 0.093) 10^{-30}$ \\
\hline & 1.75 & $(1.760 \pm 0.092) 10^{-30}$ & $(0.750 \pm 0.055) 10^{-30}$ \\
\hline & 2.25 & $(0.888 \pm 0.052) 10^{-30}$ & $(0.205 \pm 0.022) 10^{-30}$ \\
\hline \multirow[t]{5}{*}{3.00} & 0.25 & $(0.162 \pm 0.043) 10^{-30}$ & $(0.094 \pm 0.033) 10^{-30}$ \\
\hline & 0.75 & $(0.219 \pm 0.038) 10^{-30}$ & $(0.112 \pm 0.031) 10^{-30}$ \\
\hline & 1.25 & $(0.101 \pm 0.023) 10^{-30}$ & $(0.068 \pm 0.017) 10^{-30}$ \\
\hline & 1.75 & $(0.083 \pm 0.026) 10^{-30}$ & $(0.051 \pm 0.023) 10^{-30}$ \\
\hline & 2.25 & $(0.029 \pm 0.017) 10^{-30}$ & $(0.022 \pm 0.028) 10^{-30}$ \\
\hline
\end{tabular}

Table 3: 


\begin{tabular}{|c|c|c|c|}
\hline$p_{T}$ & $x_{F}$ & $E \cdot d^{3} \sigma / d p^{3} \oplus$ & $E \cdot d^{3} \sigma / d p^{3} \ominus$ \\
\hline$[\mathrm{GeV} / \mathrm{c}]$ & & {$\left[\mathrm{cm}^{2} \cdot \mathrm{c}^{3} / \mathrm{GeV}^{2}\right]$} & {$\left[\mathrm{cm}^{2} \cdot \mathrm{c}^{3} / \mathrm{GeV}^{2}\right]$} \\
\hline \multirow[t]{8}{*}{0.25} & 0.05 & $(1.114 \pm 0.005) 10^{-24}$ & $(9.913 \pm 0.043) 10^{-25}$ \\
\hline & 0.15 & $(1.230 \pm 0.006) 10^{-25}$ & $(6.700 \pm 0.040) 10^{-26}$ \\
\hline & 0.25 & $(4.987 \pm 0.042) 10^{-26}$ & $(1.156 \pm 0.015) 10^{-26}$ \\
\hline & 0.35 & $(2.615 \pm 0.029) 10^{-26}$ & $(3.606 \pm 0.083) 10^{-27}$ \\
\hline & 0.45 & $(2.007 \pm 0.026) 10^{-26}$ & $(1.441 \pm 0.056) 10^{-27}$ \\
\hline & 0.55 & $(1.770 \pm 0.029) 10^{-26}$ & $(0.613 \pm 0.039) 10^{-27}$ \\
\hline & 0.65 & $(1.490 \pm 0.034) 10^{-26}$ & $(0.199 \pm 0.019) 10^{-27}$ \\
\hline & 0.75 & $(1.473 \pm 0.071) 10^{-26}$ & $(0.527 \pm 0.095) 10^{-28}$ \\
\hline \multirow[t]{9}{*}{0.35} & 0.05 & $(6.008 \pm 0.028) 10^{-25}$ & $(5.674 \pm 0.029) 10^{-25}$ \\
\hline & 0.15 & $(8.364 \pm 0.045) 10^{-26}$ & $(5.390 \pm 0.035) 10^{-26}$ \\
\hline & 0.25 & $(3.772 \pm 0.034) 10^{-26}$ & $(1.037 \pm 0.013) 10^{-26}$ \\
\hline & 0.35 & $(2.059 \pm 0.023) 10^{-26}$ & $(2.968 \pm 0.066) 10^{-27}$ \\
\hline & 0.45 & $(1.400 \pm 0.020) 10^{-26}$ & $(1.101 \pm 0.042) 10^{-27}$ \\
\hline & 0.55 & $(1.118 \pm 0.015) 10^{-26}$ & $(0.440 \pm 0.027) 10^{-27}$ \\
\hline & 0.65 & $(1.009 \pm 0.016) 10^{-26}$ & $(0.151 \pm 0.014) 10^{-27}$ \\
\hline & 0.75 & $(0.981 \pm 0.020) 10^{-26}$ & $(0.413 \pm 0.071) 10^{-28}$ \\
\hline & 0.85 & $(1.463 \pm 0.042) 10^{-26}$ & $(0.072 \pm 0.029) 10^{-28}$ \\
\hline \multirow[t]{9}{*}{0.45} & 0.05 & $(3.249 \pm 0.019) 10^{-25}$ & $(2.703 \pm 0.015) 10^{-25}$ \\
\hline & 0.15 & $(5.290 \pm 0.032) 10^{-26}$ & $(3.409 \pm 0.023) 10^{-26}$ \\
\hline & 0.25 & $(2.498 \pm 0.022) 10^{-26}$ & $(7.608 \pm 0.099) 10^{-27}$ \\
\hline & 0.35 & $(1.486 \pm 0.018) 10^{-26}$ & $(1.917 \pm 0.046) 10^{-27}$ \\
\hline & 0.45 & $(0.952 \pm 0.013) 10^{-26}$ & $(0.747 \pm 0.028) 10^{-27}$ \\
\hline & 0.55 & $(0.807 \pm 0.013) 10^{-26}$ & $(0.308 \pm 0.017) 10^{-27}$ \\
\hline & 0.65 & $(5.907 \pm 0.098) 10^{-27}$ & $(0.917 \pm 0.088) 10^{-28}$ \\
\hline & 0.75 & $(0.561 \pm 0.011) 10^{-26}$ & $(0.308 \pm 0.048) 10^{-28}$ \\
\hline & 0.85 & $(0.738 \pm 0.020) 10^{-26}$ & $(0.097 \pm 0.028) 10^{-28}$ \\
\hline \multirow[t]{9}{*}{0.55} & 0.05 & $(1.838 \pm 0.012) 10^{-25}$ & $(1.606 \pm 0.011) 10^{-25}$ \\
\hline & 0.15 & $(3.525 \pm 0.023) 10^{-26}$ & $(2.410 \pm 0.017) 10^{-26}$ \\
\hline & 0.25 & $(1.674 \pm 0.015) 10^{-26}$ & $(6.377 \pm 0.080) 10^{-27}$ \\
\hline & 0.35 & $(1.096 \pm 0.014) 10^{-26}$ & $(1.931 \pm 0.044) 10^{-27}$ \\
\hline & 0.45 & $(7.524 \pm 0.105) 10^{-27}$ & $(0.620 \pm 0.023) 10^{-27}$ \\
\hline & 0.55 & $(5.065 \pm 0.088) 10^{-27}$ & $(0.194 \pm 0.012) 10^{-27}$ \\
\hline & 0.65 & $(3.846 \pm 0.077) 10^{-27}$ & $(0.699 \pm 0.072) 10^{-28}$ \\
\hline & 0.75 & $(3.147 \pm 0.072) 10^{-27}$ & $(0.278 \pm 0.046) 10^{-28}$ \\
\hline & 0.85 & $(3.131 \pm 0.072) 10^{-27}$ & $(0.163 \pm 0.035) 10^{-28}$ \\
\hline \multirow[t]{9}{*}{0.65} & 0.05 & $(9.954 \pm 0.064) 10^{-26}$ & $(8.378 \pm 0.060) 10^{-26}$ \\
\hline & 0.15 & $(2.247 \pm 0.015) 10^{-26}$ & $(1.415 \pm 0.011) 10^{-26}$ \\
\hline & 0.25 & $(1.168 \pm 0.012) 10^{-26}$ & $(4.382 \pm 0.062) 10^{-27}$ \\
\hline & 0.35 & $(7.244 \pm 0.088) 10^{-27}$ & $(1.435 \pm 0.033) 10^{-27}$ \\
\hline & 0.45 & $(4.985 \pm 0.069) 10^{-27}$ & $(0.429 \pm 0.017) 10^{-27}$ \\
\hline & 0.55 & $(3.414 \pm 0.059) 10^{-27}$ & $(0.180 \pm 0.012) 10^{-27}$ \\
\hline & 0.65 & $(2.212 \pm 0.048) 10^{-27}$ & $(0.475 \pm 0.055) 10^{-28}$ \\
\hline & 0.75 & $(1.709 \pm 0.040) 10^{-27}$ & $(0.115 \pm 0.026) 10^{-28}$ \\
\hline & 0.85 & $(1.764 \pm 0.048) 10^{-27}$ & $(0.051 \pm 0.019) 10^{-28}$ \\
\hline \multirow[t]{9}{*}{0.82} & 0.05 & $(3.717 \pm 0.029) 10^{-26}$ & $(3.068 \pm 0.029) 10^{-26}$ \\
\hline & 0.15 & $(9.725 \pm 0.063) 10^{-27}$ & $(5.834 \pm 0.051) 10^{-27}$ \\
\hline & 0.25 & $(4.787 \pm 0.043) 10^{-27}$ & $(1.896 \pm 0.025) 10^{-27}$ \\
\hline & 0.35 & $(3.188 \pm 0.038) 10^{-27}$ & $(0.634 \pm 0.013) 10^{-27}$ \\
\hline & 0.45 & $(2.417 \pm 0.034) 10^{-27}$ & $(2.295 \pm 0.075) 10^{-28}$ \\
\hline & 0.55 & $(1.689 \pm 0.032) 10^{-27}$ & $(0.860 \pm 0.045) 10^{-28}$ \\
\hline & 0.65 & $(1.114 \pm 0.026) 10^{-27}$ & $(0.325 \pm 0.027) 10^{-28}$ \\
\hline & 0.75 & $(0.722 \pm 0.019) 10^{-27}$ & $(0.118 \pm 0.015) 10^{-28}$ \\
\hline & 0.85 & $(0.496 \pm 0.014) 10^{-27}$ & $(0.464 \pm 0.100) 10^{-29}$ \\
\hline
\end{tabular}




\begin{tabular}{|c|c|c|c|}
\hline$p_{T}$ & $x_{F}$ & $E \cdot d^{3} \sigma / d p^{3} \oplus$ & $E \cdot d^{3} \sigma / d p^{3} \ominus$ \\
\hline$[\mathrm{GeV} / \mathrm{c}]$ & & {$\left[\mathrm{cm}^{2} \cdot \mathrm{c}^{3} / \mathrm{GeV}^{2}\right]$} & {$\left[\mathrm{cm}^{2} \cdot \mathrm{c}^{3} / \mathrm{GeV}^{2}\right]$} \\
\hline \multirow[t]{9}{*}{1.12} & 0.05 & $(7.868 \pm 0.079) 10^{-27}$ & $(6.356 \pm 0.080) 10^{-27}$ \\
\hline & 0.15 & $(2.327 \pm 0.025) 10^{-27}$ & $(1.269 \pm 0.016) 10^{-27}$ \\
\hline & 0.25 & $(1.024 \pm 0.014) 10^{-27}$ & $(4.576 \pm 0.090) 10^{-28}$ \\
\hline & 0.35 & $(0.664 \pm 0.012) 10^{-27}$ & $(1.912 \pm 0.053) 10^{-28}$ \\
\hline & 0.45 & $(4.847 \pm 0.097) 10^{-28}$ & $(0.802 \pm 0.035) 10^{-28}$ \\
\hline & 0.55 & $(3.563 \pm 0.082) 10^{-28}$ & $(0.316 \pm 0.022) 10^{-28}$ \\
\hline & 0.65 & $(2.527 \pm 0.077) 10^{-28}$ & $(0.133 \pm 0.014) 10^{-28}$ \\
\hline & 0.75 & $(1.927 \pm 0.069) 10^{-28}$ & $(0.483 \pm 0.086) 10^{-29}$ \\
\hline & 0.85 & $(1.182 \pm 0.048) 10^{-28}$ & $(0.296 \pm 0.073) 10^{-29}$ \\
\hline \multirow[t]{9}{*}{1.42} & 0.05 & $(1.934 \pm 0.028) 10^{-27}$ & $(1.555 \pm 0.028) 10^{-27}$ \\
\hline & 0.15 & $(5.943 \pm 0.092) 10^{-28}$ & $(3.500 \pm 0.072) 10^{-28}$ \\
\hline & 0.25 & $(2.470 \pm 0.054) 10^{-28}$ & $(1.111 \pm 0.034) 10^{-28}$ \\
\hline & 0.35 & $(1.413 \pm 0.039) 10^{-28}$ & $(0.519 \pm 0.023) 10^{-28}$ \\
\hline & 0.45 & $(0.895 \pm 0.033) 10^{-28}$ & $(0.300 \pm 0.017) 10^{-28}$ \\
\hline & 0.55 & $(0.691 \pm 0.028) 10^{-28}$ & $(0.183 \pm 0.014) 10^{-28}$ \\
\hline & 0.65 & $(0.440 \pm 0.022) 10^{-28}$ & $(0.112 \pm 0.011) 10^{-28}$ \\
\hline & 0.75 & $(0.378 \pm 0.020) 10^{-28}$ & $(0.427 \pm 0.067) 10^{-29}$ \\
\hline & 0.85 & $(0.230 \pm 0.016) 10^{-28}$ & $(0.219 \pm 0.049) 10^{-29}$ \\
\hline \multirow[t]{9}{*}{1.72} & 0.05 & $(0.548 \pm 0.012) 10^{-27}$ & $(0.373 \pm 0.011) 10^{-27}$ \\
\hline & 0.15 & $(1.745 \pm 0.041) 10^{-28}$ & $(0.101 \pm 0.031) 10^{-27}$ \\
\hline & 0.25 & $(0.731 \pm 0.026) 10^{-28}$ & $(0.327 \pm 0.017) 10^{-28}$ \\
\hline & 0.35 & $(0.371 \pm 0.017) 10^{-28}$ & $(0.131 \pm 0.010) 10^{-28}$ \\
\hline & 0.45 & $(0.213 \pm 0.013) 10^{-28}$ & $(0.785 \pm 0.078) 10^{-29}$ \\
\hline & 0.55 & $(0.127 \pm 0.010) 10^{-28}$ & $(0.707 \pm 0.077) 10^{-29}$ \\
\hline & 0.65 & $(0.919 \pm 0.087) 10^{-29}$ & $(0.565 \pm 0.068) 10^{-29}$ \\
\hline & 0.75 & $(0.838 \pm 0.085) 10^{-29}$ & $(0.447 \pm 0.069) 10^{-29}$ \\
\hline & 0.85 & $(0.712 \pm 0.073) 10^{-29}$ & $(0.233 \pm 0.053) 10^{-29}$ \\
\hline \multirow[t]{9}{*}{2.18} & 0.05 & $(0.709 \pm 0.023) 10^{-28}$ & $(0.579 \pm 0.022) 10^{-28}$ \\
\hline & 0.15 & $(0.303 \pm 0.010) 10^{-28}$ & $(1.911 \pm 0.074) 10^{-29}$ \\
\hline & 0.25 & $(1.263 \pm 0.059) 10^{-29}$ & $(0.548 \pm 0.034) 10^{-29}$ \\
\hline & 0.35 & $(0.702 \pm 0.042) 10^{-29}$ & $(0.273 \pm 0.025) 10^{-29}$ \\
\hline & 0.45 & $(0.348 \pm 0.027) 10^{-29}$ & $(0.118 \pm 0.016) 10^{-29}$ \\
\hline & 0.55 & $(0.257 \pm 0.025) 10^{-29}$ & $(0.166 \pm 0.022) 10^{-29}$ \\
\hline & 0.65 & $(0.111 \pm 0.016) 10^{-29}$ & $(0.175 \pm 0.024) 10^{-29}$ \\
\hline & 0.75 & $(0.086 \pm 0.015) 10^{-29}$ & $(0.285 \pm 0.042) 10^{-29}$ \\
\hline & 0.85 & $(0.145 \pm 0.023) 10^{-29}$ & $(0.309 \pm 0.048) 10^{-29}$ \\
\hline \multirow[t]{9}{*}{3.00} & 0.05 & $(0.329 \pm 0.028) 10^{-29}$ & $(0.285 \pm 0.026) 10^{-29}$ \\
\hline & 0.15 & $(0.332 \pm 0.031) 10^{-29}$ & $(0.198 \pm 0.021) 10^{-29}$ \\
\hline & 0.25 & $(0.851 \pm 0.091) 10^{-30}$ & $(0.510 \pm 0.063) 10^{-30}$ \\
\hline & 0.35 & $(0.487 \pm 0.066) 10^{-30}$ & $(0.269 \pm 0.069) 10^{-30}$ \\
\hline & 0.45 & $(0.344 \pm 0.064) 10^{-30}$ & $(0.203 \pm 0.052) 10^{-30}$ \\
\hline & 0.55 & $(0.210 \pm 0.044) 10^{-30}$ & $(0.178 \pm 0.045) 10^{-30}$ \\
\hline & 0.65 & $(0.177 \pm 0.045) 10^{-30}$ & $(0.102 \pm 0.042) 10^{-30}$ \\
\hline & 0.75 & $(0.122 \pm 0.052) 10^{-30}$ & $(0.202 \pm 0.078) 10^{-30}$ \\
\hline & 0.85 & $(0.141 \pm 0.054) 10^{-30}$ & $(0.194 \pm 0.074) 10^{-30}$ \\
\hline
\end{tabular}

Table 4: 


\begin{tabular}{|c|c|c|c|}
\hline$p_{T}$ & $x_{F}$ & $E \cdot d^{3} \sigma / d p^{3} \oplus$ & $E \cdot d^{3} \sigma / d p^{3} \ominus$ \\
\hline$[\mathrm{GeV} / \mathrm{c}]$ & & {$\left[\mathrm{cm}^{2} \cdot \mathrm{c}^{3} / \mathrm{GeV}^{2}\right]$} & {$\left[\mathrm{cm}^{2} \cdot \mathrm{c}^{3} / \mathrm{GeV}^{2}\right]$} \\
\hline \multirow[t]{9}{*}{0.25} & 0.05 & $(9.825 \pm 0.046) 10^{-25}$ & $(8.609 \pm 0.042) 10^{-25}$ \\
\hline & 0.15 & $(1.226 \pm 0.008) 10^{-25}$ & $(7.405 \pm 0.054) 10^{-26}$ \\
\hline & 0.25 & $(5.140 \pm 0.050) 10^{-26}$ & $(1.630 \pm 0.022) 10^{-26}$ \\
\hline & 0.35 & $(2.680 \pm 0.035) 10^{-26}$ & $(3.611 \pm 0.096) 10^{-27}$ \\
\hline & 0.45 & $(1.734 \pm 0.028) 10^{-26}$ & $(1.648 \pm 0.067) 10^{-27}$ \\
\hline & 0.55 & $(1.632 \pm 0.029) 10^{-26}$ & $(0.619 \pm 0.043) 10^{-27}$ \\
\hline & 0.65 & $(1.462 \pm 0.026) 10^{-26}$ & $(0.234 \pm 0.026) 10^{-27}$ \\
\hline & 0.75 & $(1.431 \pm 0.033) 10^{-26}$ & $(0.043 \pm 0.011) 10^{-27}$ \\
\hline & 0.85 & $(1.882 \pm 0.058) 10^{-26}$ & $(0.197 \pm 0.075) 10^{-28}$ \\
\hline \multirow[t]{9}{*}{0.35} & 0.05 & $(5.113 \pm 0.026) 10^{-25}$ & $(4.512 \pm 0.025) 10^{-25}$ \\
\hline & 0.15 & $(8.081 \pm 0.053) 10^{-26}$ & $(5.255 \pm 0.037) 10^{-26}$ \\
\hline & 0.25 & $(3.492 \pm 0.032) 10^{-26}$ & $(1.293 \pm 0.017) 10^{-26}$ \\
\hline & 0.35 & $(2.121 \pm 0.025) 10^{-26}$ & $(3.109 \pm 0.080) 10^{-27}$ \\
\hline & 0.45 & $(1.440 \pm 0.023) 10^{-26}$ & $(1.166 \pm 0.049) 10^{-27}$ \\
\hline & 0.55 & $(1.103 \pm 0.020) 10^{-26}$ & $(0.420 \pm 0.029) 10^{-27}$ \\
\hline & 0.65 & $(1.106 \pm 0.021) 10^{-26}$ & $(0.155 \pm 0.019) 10^{-27}$ \\
\hline & 0.75 & $(0.889 \pm 0.018) 10^{-26}$ & $(0.419 \pm 0.089) 10^{-28}$ \\
\hline & 0.85 & $(0.942 \pm 0.021) 10^{-26}$ & $(0.070 \pm 0.035) 10^{-28}$ \\
\hline \multirow[t]{9}{*}{0.45} & 0.05 & $(2.740 \pm 0.016) 10^{-25}$ & $(2.389 \pm 0.015) 10^{-25}$ \\
\hline & 0.15 & $(5.380 \pm 0.035) 10^{-26}$ & $(3.645 \pm 0.026) 10^{-26}$ \\
\hline & 0.25 & $(2.471 \pm 0.023) 10^{-26}$ & $(0.956 \pm 0.013) 10^{-26}$ \\
\hline & 0.35 & $(1.464 \pm 0.017) 10^{-26}$ & $(2.711 \pm 0.066) 10^{-27}$ \\
\hline & 0.45 & $(1.028 \pm 0.014) 10^{-26}$ & $(0.893 \pm 0.038) 10^{-27}$ \\
\hline & 0.55 & $(0.794 \pm 0.014) 10^{-26}$ & $(0.345 \pm 0.023) 10^{-27}$ \\
\hline & 0.65 & $(0.614 \pm 0.011) 10^{-26}$ & $(0.099 \pm 0.012) 10^{-27}$ \\
\hline & 0.75 & $(4.928 \pm 0.099) 10^{-27}$ & $(0.289 \pm 0.062) 10^{-28}$ \\
\hline & 0.85 & $(0.472 \pm 0.010) 10^{-26}$ & $(0.087 \pm 0.036) 10^{-28}$ \\
\hline \multirow[t]{9}{*}{0.55} & 0.05 & $(1.456 \pm 0.009) 10^{-25}$ & $(1.233 \pm 0.008) 10^{-25}$ \\
\hline & 0.15 & $(3.644 \pm 0.025) 10^{-26}$ & $(2.281 \pm 0.018) 10^{-26}$ \\
\hline & 0.25 & $(1.789 \pm 0.017) 10^{-26}$ & $(6.825 \pm 0.097) 10^{-27}$ \\
\hline & 0.35 & $(1.129 \pm 0.013) 10^{-26}$ & $(2.280 \pm 0.055) 10^{-27}$ \\
\hline & 0.45 & $(7.864 \pm 0.109) 10^{-27}$ & $(0.676 \pm 0.029) 10^{-27}$ \\
\hline & 0.55 & $(5.397 \pm 0.094) 10^{-27}$ & $(0.232 \pm 0.016) 10^{-27}$ \\
\hline & 0.65 & $(3.980 \pm 0.079) 10^{-27}$ & $(0.613 \pm 0.085) 10^{-28}$ \\
\hline & 0.75 & $(2.936 \pm 0.067) 10^{-27}$ & $(0.289 \pm 0.057) 10^{-28}$ \\
\hline & 0.85 & $(2.482 \pm 0.068) 10^{-27}$ & $(0.090 \pm 0.032) 10^{-28}$ \\
\hline \multirow[t]{9}{*}{0.65} & 0.05 & $(8.120 \pm 0.058) 10^{-26}$ & $(6.710 \pm 0.052) 10^{-26}$ \\
\hline & 0.15 & $(2.247 \pm 0.017) 10^{-26}$ & $(1.311 \pm 0.012) 10^{-26}$ \\
\hline & 0.25 & $(1.242 \pm 0.012) 10^{-26}$ & $(4.651 \pm 0.071) 10^{-27}$ \\
\hline & 0.35 & $(7.705 \pm 0.097) 10^{-27}$ & $(1.466 \pm 0.040) 10^{-27}$ \\
\hline & 0.45 & $(5.771 \pm 0.086) 10^{-27}$ & $(0.523 \pm 0.023) 10^{-27}$ \\
\hline & 0.55 & $(3.982 \pm 0.071) 10^{-27}$ & $(0.162 \pm 0.013) 10^{-27}$ \\
\hline & 0.65 & $(2.850 \pm 0.065) 10^{-27}$ & $(0.550 \pm 0.074) 10^{-28}$ \\
\hline & 0.75 & $(1.841 \pm 0.046) 10^{-27}$ & $(0.157 \pm 0.039) 10^{-28}$ \\
\hline & 0.85 & $(1.393 \pm 0.042) 10^{-27}$ & $(0.058 \pm 0.024) 10^{-28}$ \\
\hline \multirow[t]{9}{*}{0.82} & 0.05 & $(2.848 \pm 0.025) 10^{-26}$ & $(2.303 \pm 0.022) 10^{-26}$ \\
\hline & 0.15 & $(9.009 \pm 0.078) 10^{-27}$ & $(5.160 \pm 0.051) 10^{-27}$ \\
\hline & 0.25 & $(4.920 \pm 0.045) 10^{-27}$ & $(1.742 \pm 0.023) 10^{-27}$ \\
\hline & 0.35 & $(3.239 \pm 0.038) 10^{-27}$ & $(0.697 \pm 0.014) 10^{-27}$ \\
\hline & 0.45 & $(2.392 \pm 0.036) 10^{-27}$ & $(2.722 \pm 0.090) 10^{-28}$ \\
\hline & 0.55 & $(1.790 \pm 0.029) 10^{-27}$ & $(0.829 \pm 0.049) 10^{-28}$ \\
\hline & 0.65 & $(1.284 \pm 0.024) 10^{-27}$ & $(0.341 \pm 0.033) 10^{-28}$ \\
\hline & 0.75 & $(0.877 \pm 0.020) 10^{-27}$ & $(0.123 \pm 0.019) 10^{-28}$ \\
\hline & 0.85 & $(0.586 \pm 0.017) 10^{-27}$ & $(0.049 \pm 0.012) 10^{-28}$ \\
\hline
\end{tabular}




\begin{tabular}{|c|c|c|c|}
\hline$p_{T}$ & $x_{F}$ & $E \cdot d^{3} \sigma / d p^{3} a \oplus$ & $E \cdot d^{3} \sigma / d p^{3} \ominus$ \\
\hline$[\mathrm{GeV} / \mathrm{c}]$ & & {$\left[\mathrm{cm}^{2} \cdot \mathrm{c}^{3} / \mathrm{GeV}^{2}\right]$} & {$\left[\mathrm{cm}^{2} \cdot \mathrm{c}^{3} / \mathrm{GeV}^{2}\right]$} \\
\hline \multirow[t]{9}{*}{1.12} & 0.05 & $(5.722 \pm 0.074) 10^{-27}$ & $(4.344 \pm 0.064) 10^{-27}$ \\
\hline & 0.15 & $(2.021 \pm 0.028) 10^{-27}$ & $(1.122 \pm 0.019) 10^{-27}$ \\
\hline & 0.25 & $(0.948 \pm 0.016) 10^{-27}$ & $(3.852 \pm 0.092) 10^{-28}$ \\
\hline & 0.35 & $(0.629 \pm 0.012) 10^{-27}$ & $(1.615 \pm 0.056) 10^{-28}$ \\
\hline & 0.45 & $(0.468 \pm 0.010) 10^{-27}$ & $(0.682 \pm 0.036) 10^{-28}$ \\
\hline & 0.55 & $(0.376 \pm 0.010) 10^{-27}$ & $(0.344 \pm 0.026) 10^{-28}$ \\
\hline & 0.65 & $(2.555 \pm 0.081) 10^{-28}$ & $(0.115 \pm 0.015) 10^{-28}$ \\
\hline & 0.75 & $(1.650 \pm 0.062) 10^{-28}$ & $(0.505 \pm 0.096) 10^{-29}$ \\
\hline & 0.85 & $(1.216 \pm 0.056) 10^{-28}$ & $(0.174 \pm 0.071) 10^{-29}$ \\
\hline \multirow[t]{9}{*}{1.42} & 0.05 & $(1.279 \pm 0.028) 10^{-27}$ & $(1.003 \pm 0.026) 10^{-27}$ \\
\hline & 0.15 & $(0.490 \pm 0.012) 10^{-27}$ & $(3.131 \pm 0.095) 10^{-28}$ \\
\hline & 0.25 & $(2.503 \pm 0.069) 10^{-28}$ & $(0.939 \pm 0.038) 10^{-28}$ \\
\hline & 0.35 & $(1.241 \pm 0.043) 10^{-28}$ & $(0.348 \pm 0.023) 10^{-28}$ \\
\hline & 0.45 & $(0.870 \pm 0.036) 10^{-28}$ & $(0.188 \pm 0.017) 10^{-28}$ \\
\hline & 0.55 & $(0.664 \pm 0.032) 10^{-28}$ & $(0.082 \pm 0.011) 10^{-28}$ \\
\hline & 0.65 & $(0.458 \pm 0.027) 10^{-28}$ & $(0.556 \pm 0.088) 10^{-29}$ \\
\hline & 0.75 & $(0.302 \pm 0.022) 10^{-28}$ & $(0.458 \pm 0.087) 10^{-29}$ \\
\hline & 0.85 & $(0.200 \pm 0.017) 10^{-28}$ & $(0.207 \pm 0.054) 10^{-29}$ \\
\hline \multirow[t]{9}{*}{1.72} & 0.05 & $(0.288 \pm 0.011) 10^{-27}$ & $(2.206 \pm 0.096) 10^{-28}$ \\
\hline & 0.15 & $(1.416 \pm 0.055) 10^{-28}$ & $(0.931 \pm 0.045) 10^{-28}$ \\
\hline & 0.25 & $(0.632 \pm 0.028) 10^{-28}$ & $(0.253 \pm 0.017) 10^{-28}$ \\
\hline & 0.35 & $(0.400 \pm 0.023) 10^{-28}$ & $(0.106 \pm 0.011) 10^{-28}$ \\
\hline & 0.45 & $(0.231 \pm 0.016) 10^{-28}$ & $(0.528 \pm 0.080) 10^{-29}$ \\
\hline & 0.55 & $(0.135 \pm 0.012) 10^{-28}$ & $(0.178 \pm 0.043) 10^{-29}$ \\
\hline & 0.65 & $(0.875 \pm 0.099) 10^{-29}$ & $(0.166 \pm 0.046) 10^{-29}$ \\
\hline & 0.75 & $(0.413 \pm 0.070) 10^{-29}$ & $(0.238 \pm 0.054) 10^{-29}$ \\
\hline & 0.85 & $(0.202 \pm 0.059) 10^{-29}$ & $(0.245 \pm 0.058) 10^{-29}$ \\
\hline \multirow[t]{9}{*}{2.18} & 0.05 & $(0.404 \pm 0.019) 10^{-28}$ & $(0.214 \pm 0.018) 10^{-28}$ \\
\hline & 0.15 & $(0.245 \pm 0.012) 10^{-28}$ & $(0.163 \pm 0.011) 10^{-28}$ \\
\hline & 0.25 & $(0.956 \pm 0.055) 10^{-29}$ & $(0.576 \pm 0.046) 10^{-29}$ \\
\hline & 0.35 & $(0.520 \pm 0.041) 10^{-29}$ & $(0.220 \pm 0.026) 10^{-29}$ \\
\hline & 0.45 & $(0.358 \pm 0.032) 10^{-29}$ & $(0.087 \pm 0.015) 10^{-29}$ \\
\hline & 0.55 & $(0.244 \pm 0.030) 10^{-29}$ & $(0.062 \pm 0.015) 10^{-29}$ \\
\hline & 0.65 & $(0.125 \pm 0.021) 10^{-29}$ & $(0.301 \pm 0.079) 10^{-29}$ \\
\hline & 0.75 & $(0.117 \pm 0.024) 10^{-29}$ & $(0.098 \pm 0.014) 10^{-29}$ \\
\hline & 0.85 & $(0.130 \pm 0.033) 10^{-29}$ & $(0.164 \pm 0.022) 10^{-29}$ \\
\hline \multirow[t]{8}{*}{3.00} & 0.05 & $(0.168 \pm 0.022) 10^{-29}$ & $(0.157 \pm 0.024) 10^{-29}$ \\
\hline & 0.15 & $(0.187 \pm 0.027) 10^{-29}$ & $(0.164 \pm 0.099) 10^{-29}$ \\
\hline & 0.25 & $(0.131 \pm 0.018) 10^{-29}$ & $(0.087 \pm 0.018) 10^{-29}$ \\
\hline & 0.35 & $(0.539 \pm 0.097) 10^{-30}$ & $(0.165 \pm 0.040) 10^{-30}$ \\
\hline & 0.45 & $(0.394 \pm 0.100) 10^{-30}$ & $(0.068 \pm 0.024) 10^{-30}$ \\
\hline & 0.55 & $(0.135 \pm 0.042) 10^{-30}$ & $(0.106 \pm 0.053) 10^{-30}$ \\
\hline & 0.65 & $(0.101 \pm 0.030) 10^{-30}$ & $(0.169 \pm 0.086) 10^{-30}$ \\
\hline & 0.75 & $(0.205 \pm 0.069) 10^{-30}$ & $(0.086 \pm 0.050) 10^{-30}$ \\
\hline
\end{tabular}

Table 5: 


\begin{tabular}{|c|c|c|c|}
\hline$p_{T}$ & $x_{F}$ & $E \cdot d^{3} \sigma / d p^{3} \oplus$ & $E \cdot d^{3} \sigma / d p^{3} \ominus$ \\
\hline$[\mathrm{GeV} / \mathrm{c}]$ & & {$\left[\mathrm{cm}^{2} \cdot \mathrm{c}^{3} / \mathrm{GeV}^{2}\right]$} & {$\left[\mathrm{cm}^{2} \cdot \mathrm{c}^{3} / \mathrm{GeV}^{2}\right]$} \\
\hline \multirow[t]{9}{*}{0.25} & 0.05 & $(8.125 \pm 0.042) 10^{-25}$ & $(7.475 \pm 0.043) 10^{-25}$ \\
\hline & 0.15 & $(1.228 \pm 0.007) 10^{-25}$ & $(7.890 \pm 0.054) 10^{-26}$ \\
\hline & 0.25 & $(5.275 \pm 0.048) 10^{-26}$ & $(1.896 \pm 0.024) 10^{-26}$ \\
\hline & 0.35 & $(3.090 \pm 0.038) 10^{-26}$ & $(0.522 \pm 0.012) 10^{-26}$ \\
\hline & 0.45 & $(2.024 \pm 0.030) 10^{-26}$ & $(1.968 \pm 0.071) 10^{-27}$ \\
\hline & 0.55 & $(1.879 \pm 0.034) 10^{-26}$ & $(0.758 \pm 0.043) 10^{-27}$ \\
\hline & 0.65 & $(1.668 \pm 0.029) 10^{-26}$ & $(0.271 \pm 0.025) 10^{-27}$ \\
\hline & 0.75 & $(1.641 \pm 0.031) 10^{-26}$ & $(0.071 \pm 0.013) 10^{-27}$ \\
\hline & 0.85 & $(2.166 \pm 0.047) 10^{-26}$ & $(0.041 \pm 0.011) 10^{-27}$ \\
\hline \multirow[t]{9}{*}{0.35} & 0.05 & $(4.302 \pm 0.023) 10^{-25}$ & $(3.797 \pm 0.023) 10^{-25}$ \\
\hline & 0.15 & $(9.050 \pm 0.049) 10^{-26}$ & $(5.663 \pm 0.036) 10^{-26}$ \\
\hline & 0.25 & $(3.774 \pm 0.030) 10^{-26}$ & $(1.474 \pm 0.018) 10^{-26}$ \\
\hline & 0.35 & $(2.260 \pm 0.023) 10^{-26}$ & $(4.246 \pm 0.087) 10^{-27}$ \\
\hline & 0.45 & $(1.611 \pm 0.021) 10^{-26}$ & $(1.424 \pm 0.052) 10^{-27}$ \\
\hline & 0.55 & $(1.289 \pm 0.018) 10^{-26}$ & $(0.506 \pm 0.030) 10^{-27}$ \\
\hline & 0.65 & $(1.032 \pm 0.017) 10^{-26}$ & $(0.188 \pm 0.018) 10^{-27}$ \\
\hline & 0.75 & $(0.928 \pm 0.018) 10^{-26}$ & $(0.652 \pm 0.105) 10^{-28}$ \\
\hline & 0.85 & $(1.017 \pm 0.023) 10^{-26}$ & $(0.213 \pm 0.062) 10^{-28}$ \\
\hline \multirow[t]{9}{*}{0.45} & 0.05 & $(2.295 \pm 0.014) 10^{-25}$ & $(1.950 \pm 0.014) 10^{-25}$ \\
\hline & 0.15 & $(6.155 \pm 0.037) 10^{-26}$ & $(3.579 \pm 0.025) 10^{-26}$ \\
\hline & 0.25 & $(2.834 \pm 0.021) 10^{-26}$ & $(1.055 \pm 0.012) 10^{-26}$ \\
\hline & 0.35 & $(1.765 \pm 0.018) 10^{-26}$ & $(3.474 \pm 0.070) 10^{-27}$ \\
\hline & 0.45 & $(1.219 \pm 0.015) 10^{-26}$ & $(1.136 \pm 0.039) 10^{-27}$ \\
\hline & 0.55 & $(0.942 \pm 0.013) 10^{-26}$ & $(0.389 \pm 0.024) 10^{-27}$ \\
\hline & 0.65 & $(0.742 \pm 0.013) 10^{-26}$ & $(0.142 \pm 0.014) 10^{-27}$ \\
\hline & 0.75 & $(5.448 \pm 0.098) 10^{-27}$ & $(0.295 \pm 0.063) 10^{-28}$ \\
\hline & 0.85 & $(0.486 \pm 0.010) 10^{-26}$ & $(0.147 \pm 0.046) 10^{-28}$ \\
\hline \multirow[t]{9}{*}{0.55} & 0.05 & $(1.217 \pm 0.009) 10^{-25}$ & $(1.041 \pm 0.009) 10^{-25}$ \\
\hline & 0.15 & $(3.815 \pm 0.026) 10^{-26}$ & $(2.278 \pm 0.019) 10^{-26}$ \\
\hline & 0.25 & $(1.843 \pm 0.014) 10^{-26}$ & $(6.747 \pm 0.082) 10^{-27}$ \\
\hline & 0.35 & $(1.262 \pm 0.014) 10^{-26}$ & $(2.592 \pm 0.051) 10^{-27}$ \\
\hline & 0.45 & $(0.884 \pm 0.010) 10^{-26}$ & $(0.849 \pm 0.030) 10^{-27}$ \\
\hline & 0.55 & $(6.584 \pm 0.091) 10^{-27}$ & $(0.325 \pm 0.019) 10^{-27}$ \\
\hline & 0.65 & $(4.722 \pm 0.077) 10^{-27}$ & $(0.821 \pm 0.096) 10^{-28}$ \\
\hline & 0.75 & $(3.558 \pm 0.068) 10^{-27}$ & $(0.316 \pm 0.061) 10^{-28}$ \\
\hline & 0.85 & $(3.445 \pm 0.079) 10^{-27}$ & $(0.174 \pm 0.050) 10^{-28}$ \\
\hline \multirow[t]{9}{*}{0.65} & 0.05 & $(6.659 \pm 0.055) 10^{-26}$ & $(5.331 \pm 0.055) 10^{-26}$ \\
\hline & 0.15 & $(2.186 \pm 0.018) 10^{-26}$ & $(1.302 \pm 0.013) 10^{-26}$ \\
\hline & 0.25 & $(1.192 \pm 0.012) 10^{-26}$ & $(4.445 \pm 0.062) 10^{-27}$ \\
\hline & 0.35 & $(8.093 \pm 0.087) 10^{-27}$ & $(1.731 \pm 0.039) 10^{-27}$ \\
\hline & 0.45 & $(6.241 \pm 0.084) 10^{-27}$ & $(0.631 \pm 0.023) 10^{-27}$ \\
\hline & 0.55 & $(4.384 \pm 0.064) 10^{-27}$ & $(0.224 \pm 0.014) 10^{-27}$ \\
\hline & 0.65 & $(3.180 \pm 0.055) 10^{-27}$ & $(0.871 \pm 0.089) 10^{-28}$ \\
\hline & 0.75 & $(2.232 \pm 0.046) 10^{-27}$ & $(0.234 \pm 0.046) 10^{-28}$ \\
\hline & 0.85 & $(1.994 \pm 0.049) 10^{-27}$ & $(0.074 \pm 0.028) 10^{-28}$ \\
\hline \multirow[t]{9}{*}{0.82} & 0.05 & $(2.204 \pm 0.021) 10^{-26}$ & $(0.170 \pm 0.018) 10^{-25}$ \\
\hline & 0.15 & $(8.064 \pm 0.081) 10^{-27}$ & $(0.486 \pm 0.057) 10^{-26}$ \\
\hline & 0.25 & $(4.644 \pm 0.048) 10^{-27}$ & $(0.178 \pm 0.024) 10^{-26}$ \\
\hline & 0.35 & $(3.361 \pm 0.038) 10^{-27}$ & $(0.710 \pm 0.014) 10^{-27}$ \\
\hline & 0.45 & $(2.682 \pm 0.032) 10^{-27}$ & $(2.908 \pm 0.083) 10^{-28}$ \\
\hline & 0.55 & $(1.941 \pm 0.029) 10^{-27}$ & $(1.068 \pm 0.048) 10^{-28}$ \\
\hline & 0.65 & $(1.436 \pm 0.025) 10^{-27}$ & $(0.399 \pm 0.030) 10^{-28}$ \\
\hline & 0.75 & $(0.972 \pm 0.020) 10^{-27}$ & $(0.135 \pm 0.017) 10^{-28}$ \\
\hline & 0.85 & $(0.743 \pm 0.017) 10^{-27}$ & $(0.050 \pm 0.011) 10^{-28}$ \\
\hline
\end{tabular}




\begin{tabular}{|c|c|c|c|}
\hline$p_{T}$ & $x_{F}$ & $E \cdot d^{3} \sigma / d p^{3} \oplus$ & $E \cdot d^{3} \sigma / d p^{3} \ominus$ \\
\hline$[\mathrm{GeV} / \mathrm{c}]$ & & {$\left[\mathrm{cm}^{2} \cdot \mathrm{c}^{3} / \mathrm{GeV}^{2}\right]$} & {$\left[\mathrm{cm}^{2} \cdot \mathrm{c}^{3} / \mathrm{GeV}^{2}\right]$} \\
\hline \multirow[t]{9}{*}{1.12} & 0.05 & $(3.858 \pm 0.064) 10^{-27}$ & $(2.876 \pm 0.053) 10^{-27}$ \\
\hline & 0.15 & $(1.867 \pm 0.032) 10^{-27}$ & $(1.096 \pm 0.022) 10^{-27}$ \\
\hline & 0.25 & $(0.959 \pm 0.016) 10^{-27}$ & $(3.611 \pm 0.085) 10^{-28}$ \\
\hline & 0.35 & $(0.675 \pm 0.013) 10^{-27}$ & $(1.554 \pm 0.053) 10^{-28}$ \\
\hline & 0.45 & $(0.518 \pm 0.011) 10^{-27}$ & $(0.641 \pm 0.032) 10^{-28}$ \\
\hline & 0.55 & $(3.729 \pm 0.089) 10^{-28}$ & $(0.248 \pm 0.020) 10^{-28}$ \\
\hline & 0.65 & $(2.946 \pm 0.078) 10^{-28}$ & $(0.095 \pm 0.012) 10^{-28}$ \\
\hline & 0.75 & $(1.907 \pm 0.066) 10^{-28}$ & $(0.539 \pm 0.093) 10^{-29}$ \\
\hline & 0.85 & $(1.288 \pm 0.051) 10^{-28}$ & $(0.362 \pm 0.078) 10^{-29}$ \\
\hline \multirow[t]{9}{*}{1.42} & 0.05 & $(0.833 \pm 0.024) 10^{-27}$ & $(0.555 \pm 0.019) 10^{-27}$ \\
\hline & 0.15 & $(0.468 \pm 0.012) 10^{-27}$ & $(2.447 \pm 0.084) 10^{-28}$ \\
\hline & 0.25 & $(2.123 \pm 0.058) 10^{-28}$ & $(0.906 \pm 0.037) 10^{-28}$ \\
\hline & 0.35 & $(1.551 \pm 0.051) 10^{-28}$ & $(0.330 \pm 0.020) 10^{-28}$ \\
\hline & 0.45 & $(1.038 \pm 0.038) 10^{-28}$ & $(0.126 \pm 0.013) 10^{-28}$ \\
\hline & 0.55 & $(0.664 \pm 0.030) 10^{-28}$ & $(0.471 \pm 0.071) 10^{-29}$ \\
\hline & 0.65 & $(0.513 \pm 0.026) 10^{-28}$ & $(0.403 \pm 0.067) 10^{-29}$ \\
\hline & 0.75 & $(0.303 \pm 0.020) 10^{-28}$ & $(0.135 \pm 0.038) 10^{-29}$ \\
\hline & 0.85 & $(0.243 \pm 0.019) 10^{-28}$ & $(0.123 \pm 0.039) 10^{-29}$ \\
\hline \multirow[t]{9}{*}{1.72} & 0.05 & $(1.910 \pm 0.097) 10^{-28}$ & $(1.063 \pm 0.067) 10^{-28}$ \\
\hline & 0.15 & $(1.189 \pm 0.055) 10^{-28}$ & $(0.589 \pm 0.036) 10^{-28}$ \\
\hline & 0.25 & $(0.570 \pm 0.029) 10^{-28}$ & $(0.254 \pm 0.017) 10^{-28}$ \\
\hline & 0.35 & $(0.397 \pm 0.020) 10^{-28}$ & $(0.123 \pm 0.011) 10^{-28}$ \\
\hline & 0.45 & $(0.257 \pm 0.017) 10^{-28}$ & $(0.406 \pm 0.061) 10^{-29}$ \\
\hline & 0.55 & $(0.138 \pm 0.012) 10^{-28}$ & $(0.140 \pm 0.032) 10^{-29}$ \\
\hline & 0.65 & $(0.137 \pm 0.010) 10^{-28}$ & $(0.107 \pm 0.029) 10^{-29}$ \\
\hline & 0.75 & $(0.714 \pm 0.080) 10^{-29}$ & $(0.042 \pm 0.021) 10^{-29}$ \\
\hline & 0.85 & $(0.426 \pm 0.066) 10^{-29}$ & $(0.058 \pm 0.022) 10^{-29}$ \\
\hline \multirow[t]{9}{*}{2.18} & 0.05 & $(0.201 \pm 0.014) 10^{-28}$ & $(0.160 \pm 0.013) 10^{-28}$ \\
\hline & 0.15 & $(0.214 \pm 0.016) 10^{-28}$ & $(0.203 \pm 0.018) 10^{-28}$ \\
\hline & 0.25 & $(0.867 \pm 0.067) 10^{-29}$ & $(0.579 \pm 0.057) 10^{-29}$ \\
\hline & 0.35 & $(0.574 \pm 0.048) 10^{-29}$ & $(0.281 \pm 0.035) 10^{-29}$ \\
\hline & 0.45 & $(0.392 \pm 0.041) 10^{-29}$ & $(0.100 \pm 0.026) 10^{-28}$ \\
\hline & 0.55 & $(0.356 \pm 0.046) 10^{-29}$ & $(0.134 \pm 0.037) 10^{-29}$ \\
\hline & 0.65 & $(0.166 \pm 0.021) 10^{-29}$ & $(0.313 \pm 0.090) 10^{-29}$ \\
\hline & 0.75 & $(0.117 \pm 0.017) 10^{-29}$ & $(0.046 \pm 0.011) 10^{-29}$ \\
\hline & 0.85 & $(0.097 \pm 0.015) 10^{-29}$ & $(0.120 \pm 0.052) 10^{-29}$ \\
\hline \multirow[t]{9}{*}{3.00} & 0.05 & $(0.087 \pm 0.019) 10^{-29}$ & $(0.034 \pm 0.012) 10^{-29}$ \\
\hline & 0.15 & $(0.082 \pm 0.015) 10^{-29}$ & $(0.058 \pm 0.013) 10^{-29}$ \\
\hline & 0.25 & $(0.074 \pm 0.016) 10^{-29}$ & $(0.049 \pm 0.013) 10^{-29}$ \\
\hline & 0.35 & $(0.270 \pm 0.066) 10^{-30}$ & $(0.029 \pm 0.010) 10^{-29}$ \\
\hline & 0.45 & $(0.101 \pm 0.046) 10^{-30}$ & $(0.133 \pm 0.062) 10^{-30}$ \\
\hline & 0.55 & $(0.149 \pm 0.059) 10^{-30}$ & $(0.160 \pm 0.067) 10^{-30}$ \\
\hline & 0.65 & $(0.311 \pm 0.096) 10^{-30}$ & $(0.216 \pm 0.062) 10^{-30}$ \\
\hline & 0.75 & $(0.106 \pm 0.031) 10^{-30}$ & $(0.104 \pm 0.055) 10^{-30}$ \\
\hline & 0.85 & $(0.158 \pm 0.053) 10^{-30}$ & $(0.278 \pm 0.153) 10^{-30}$ \\
\hline
\end{tabular}

Table 6: 


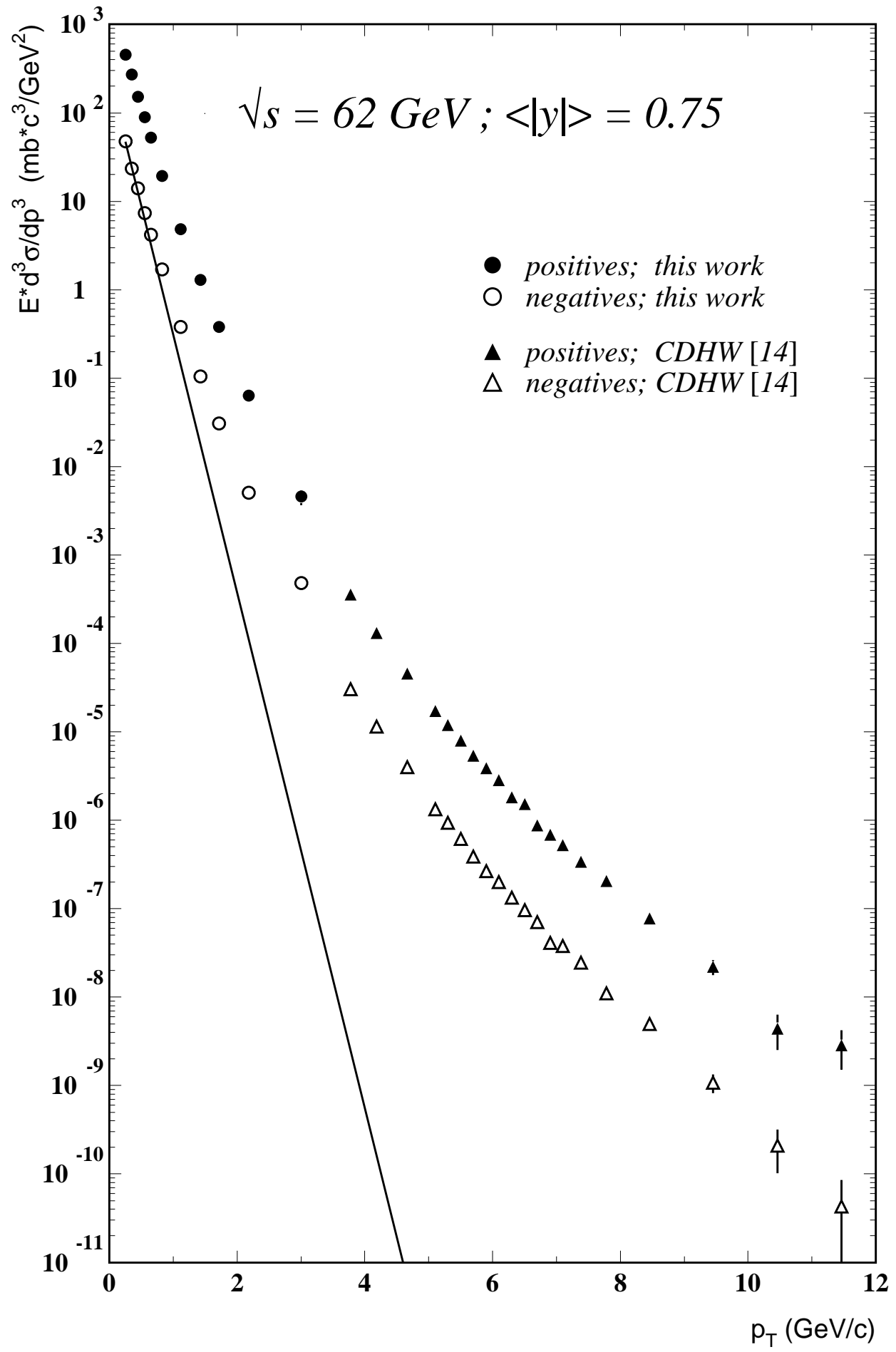

Fig. 1 


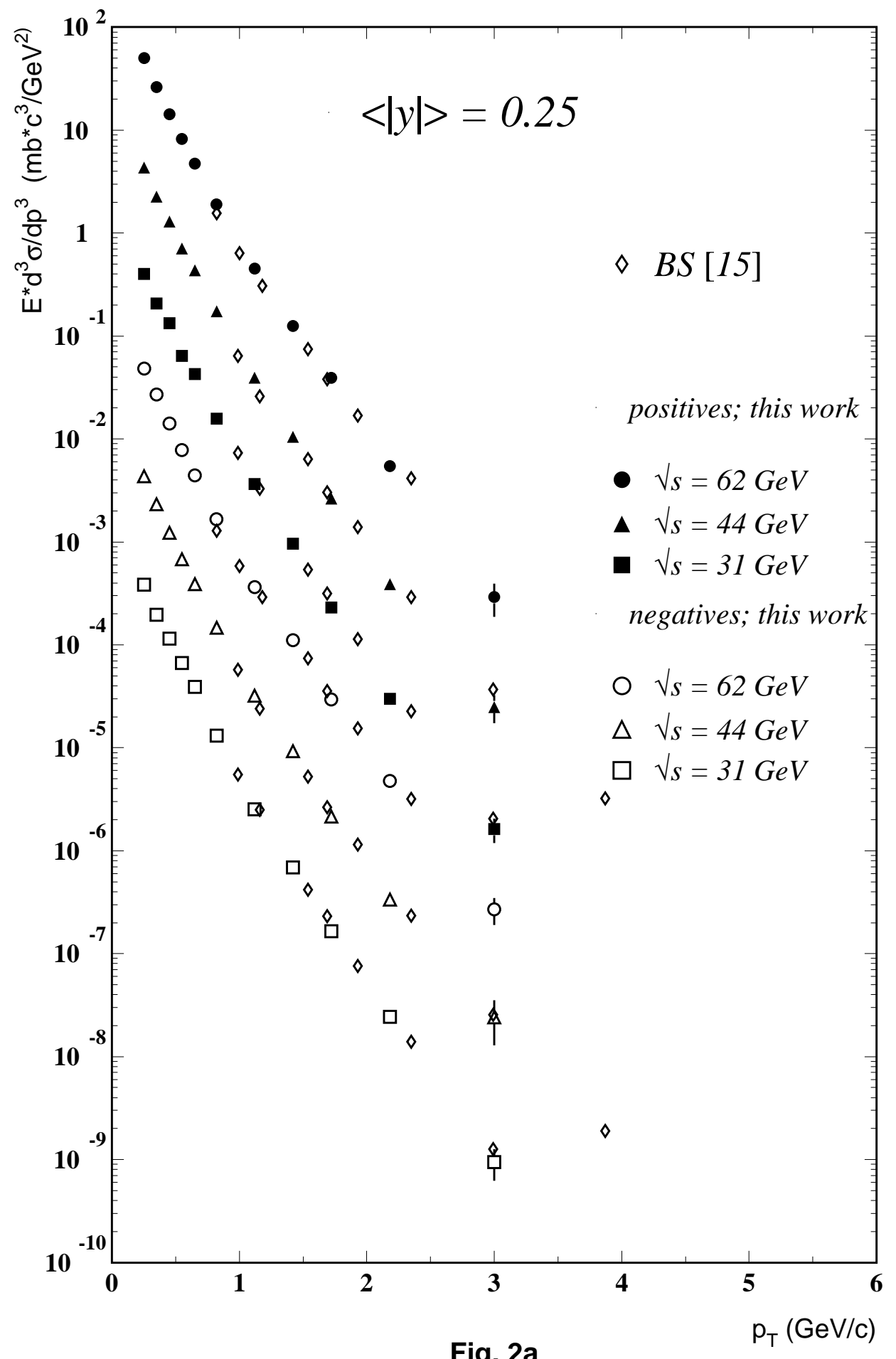

Fig. 2a 


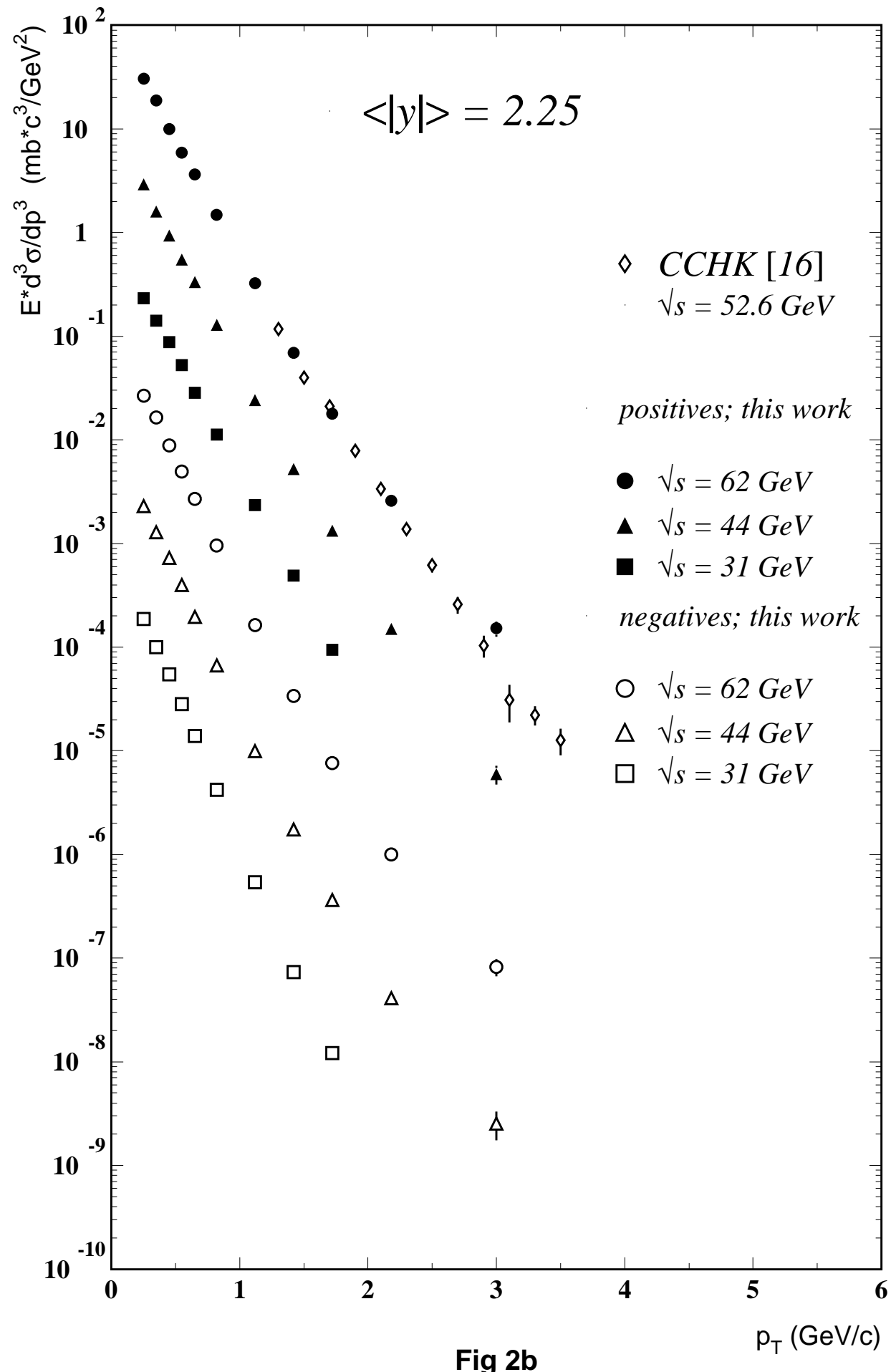

Fig $2 b$ 


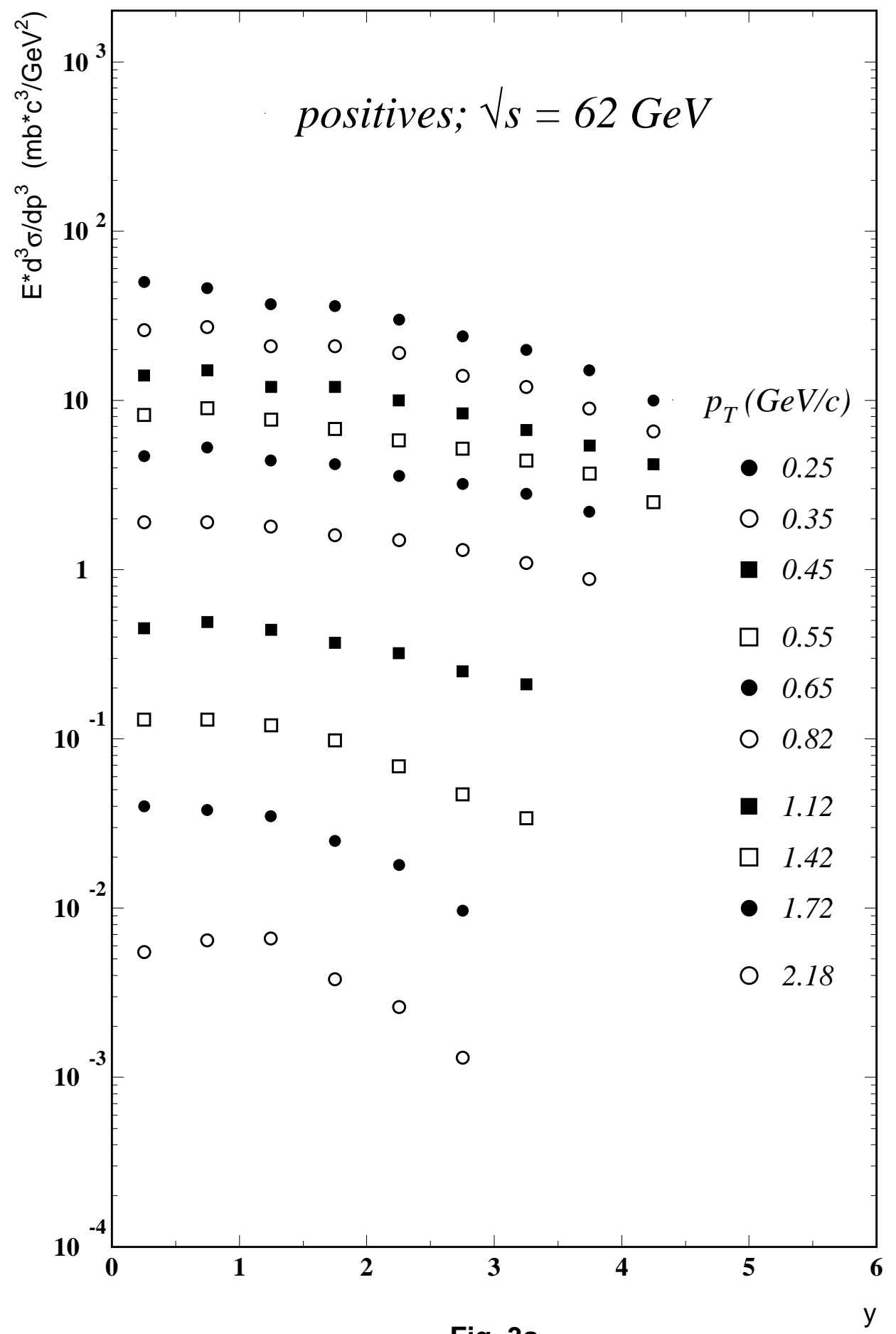

Fig. 3a 


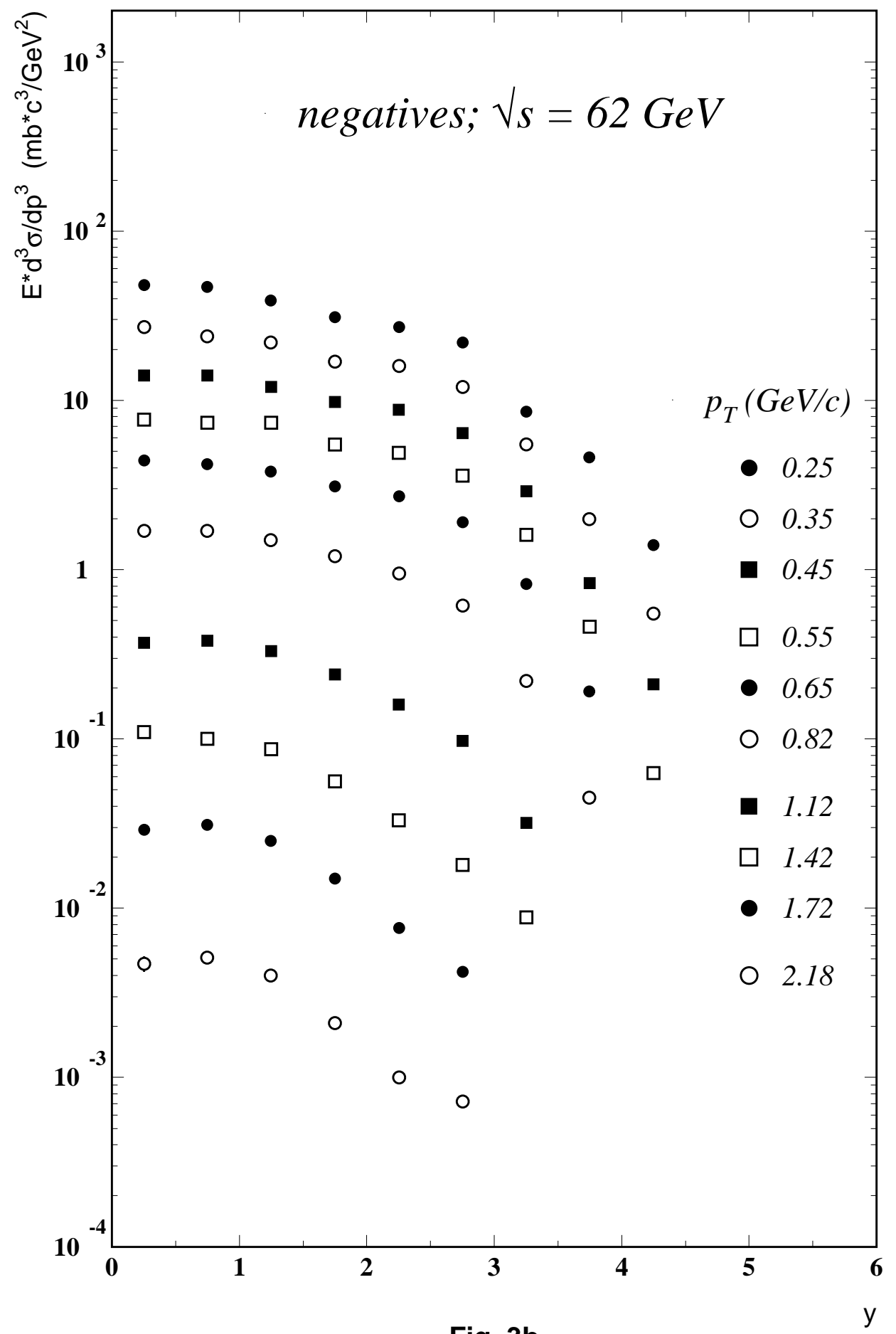

Fig. 3b 


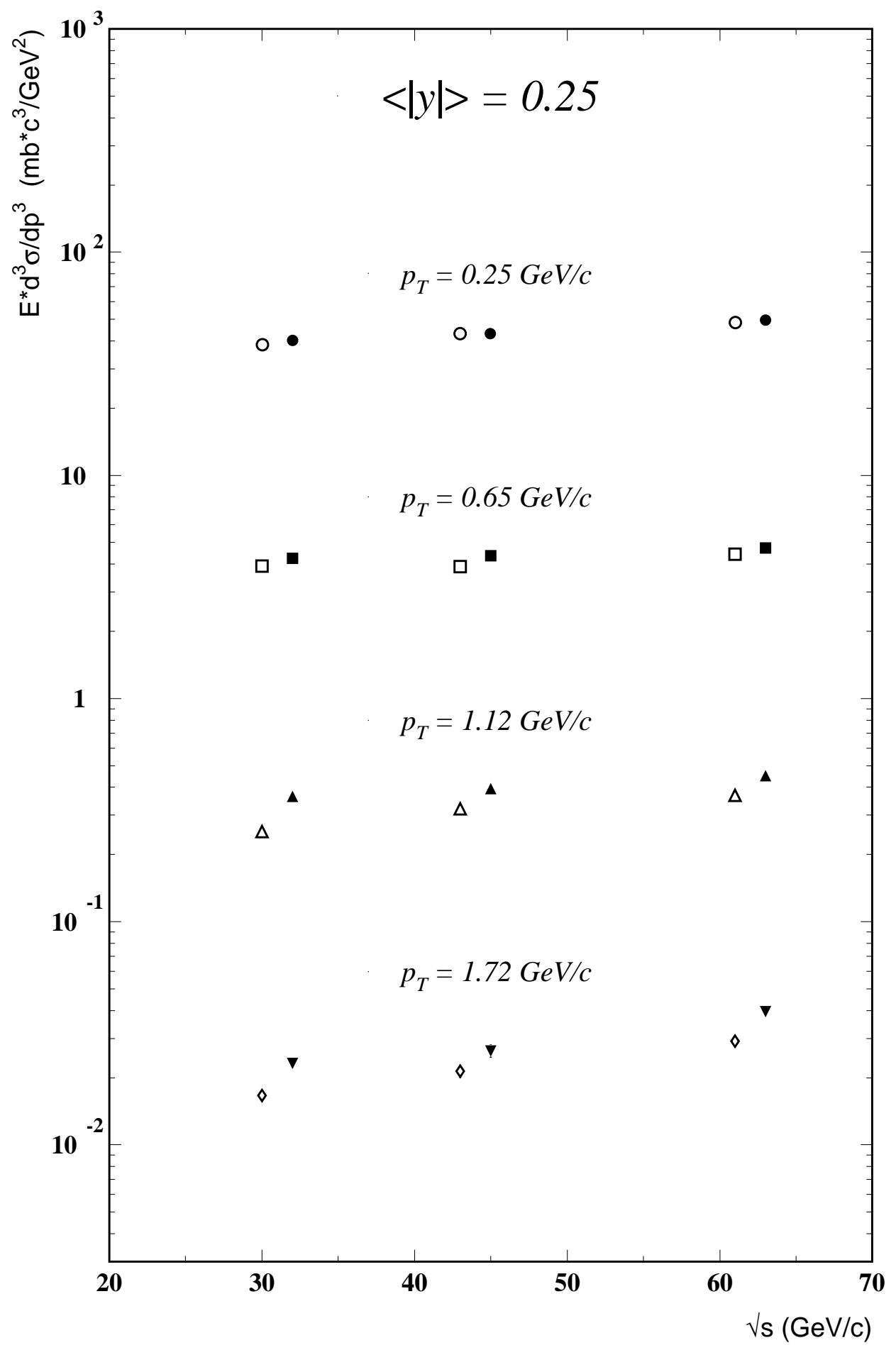

Fig. 4 


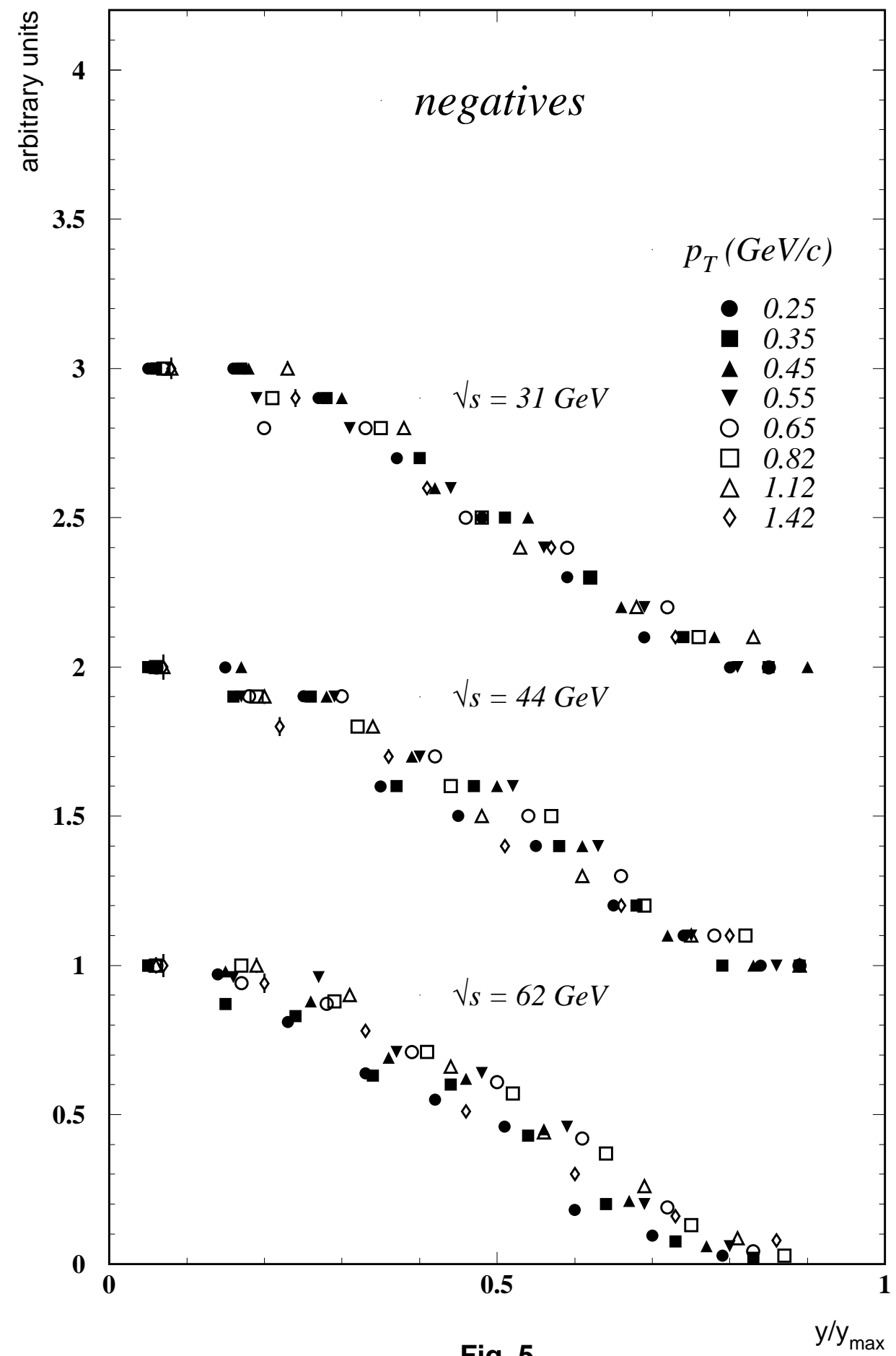

Fig. 5 


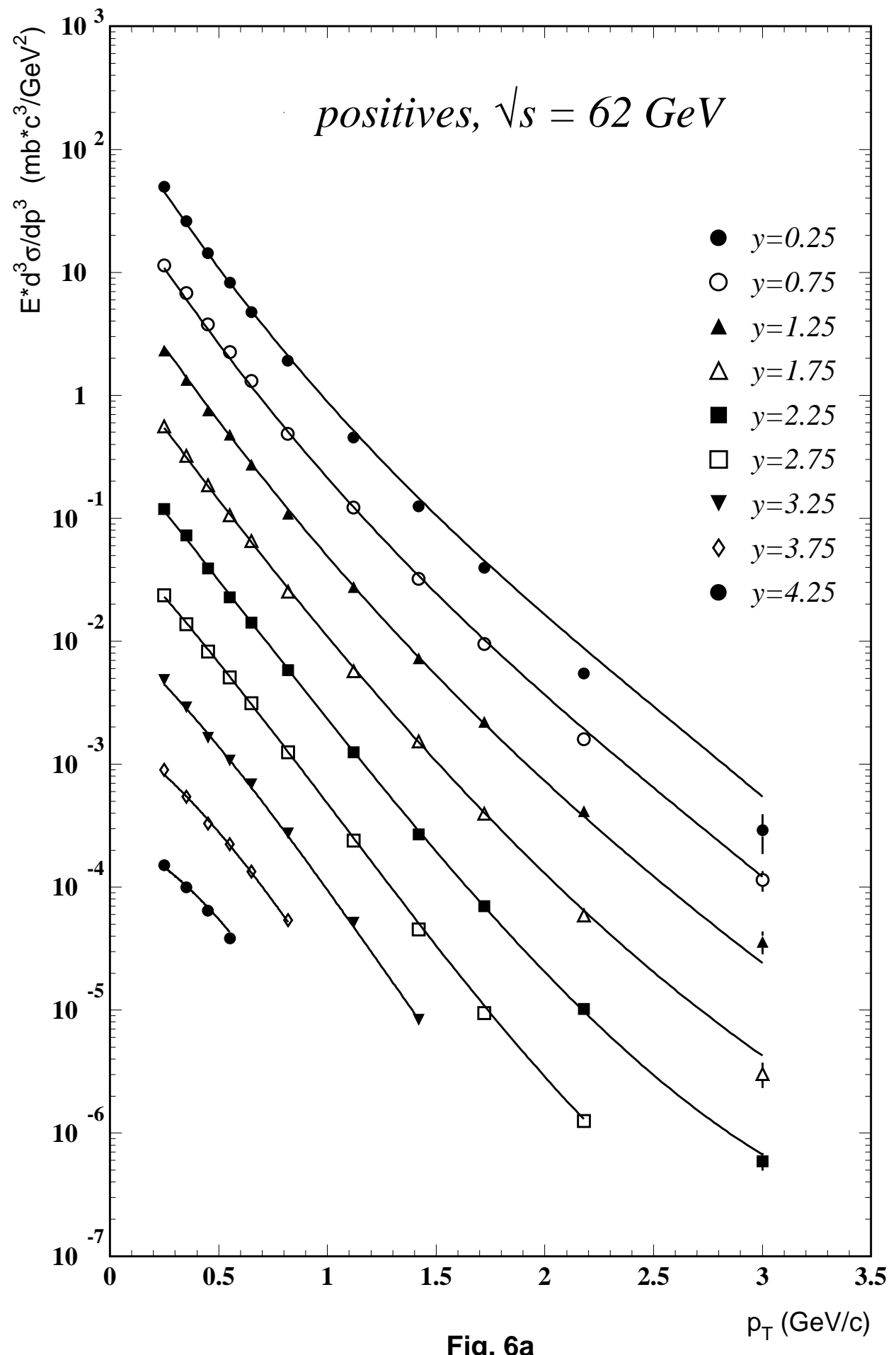

Fig. 6a 


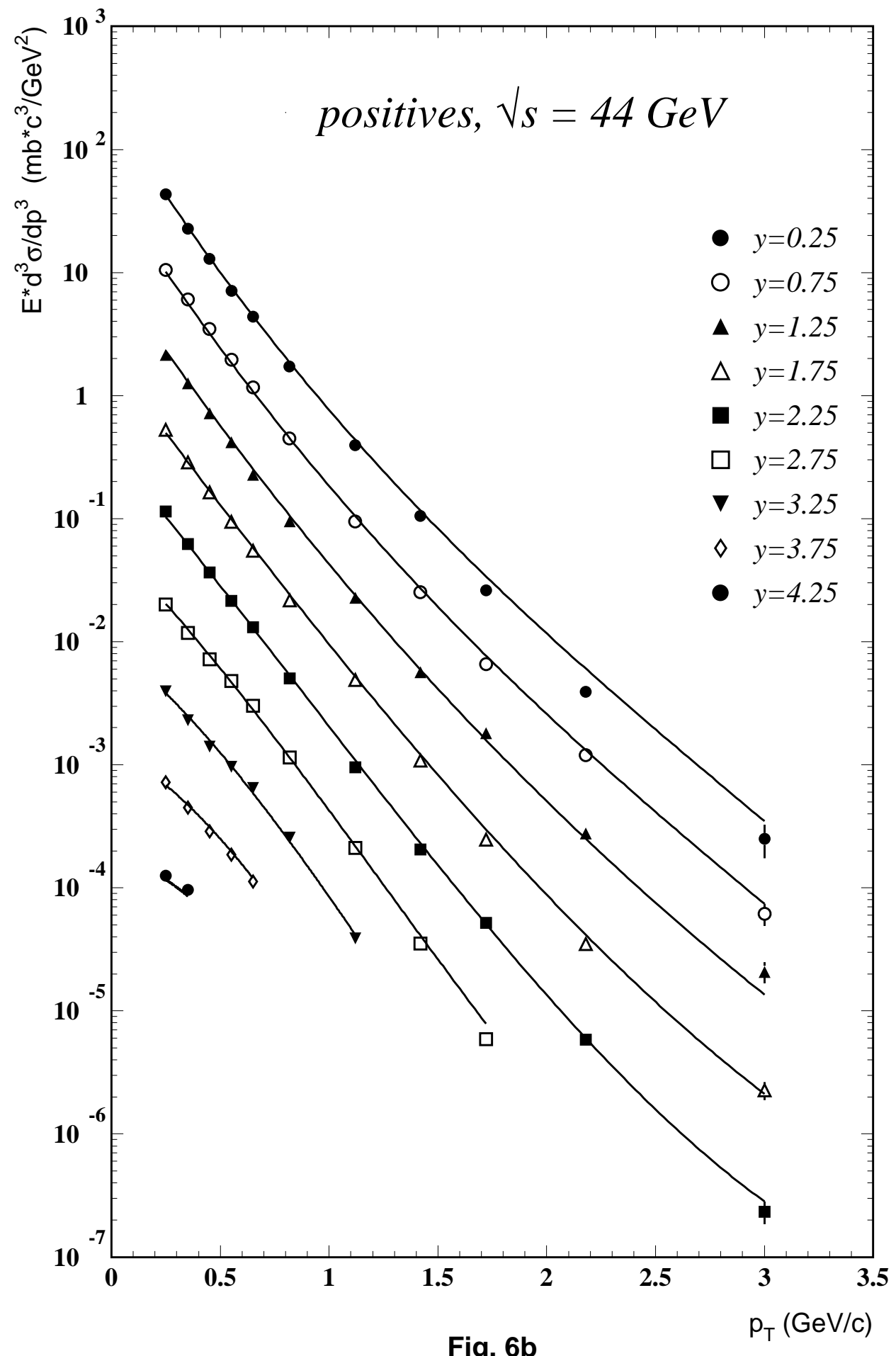

Fig. 6b 


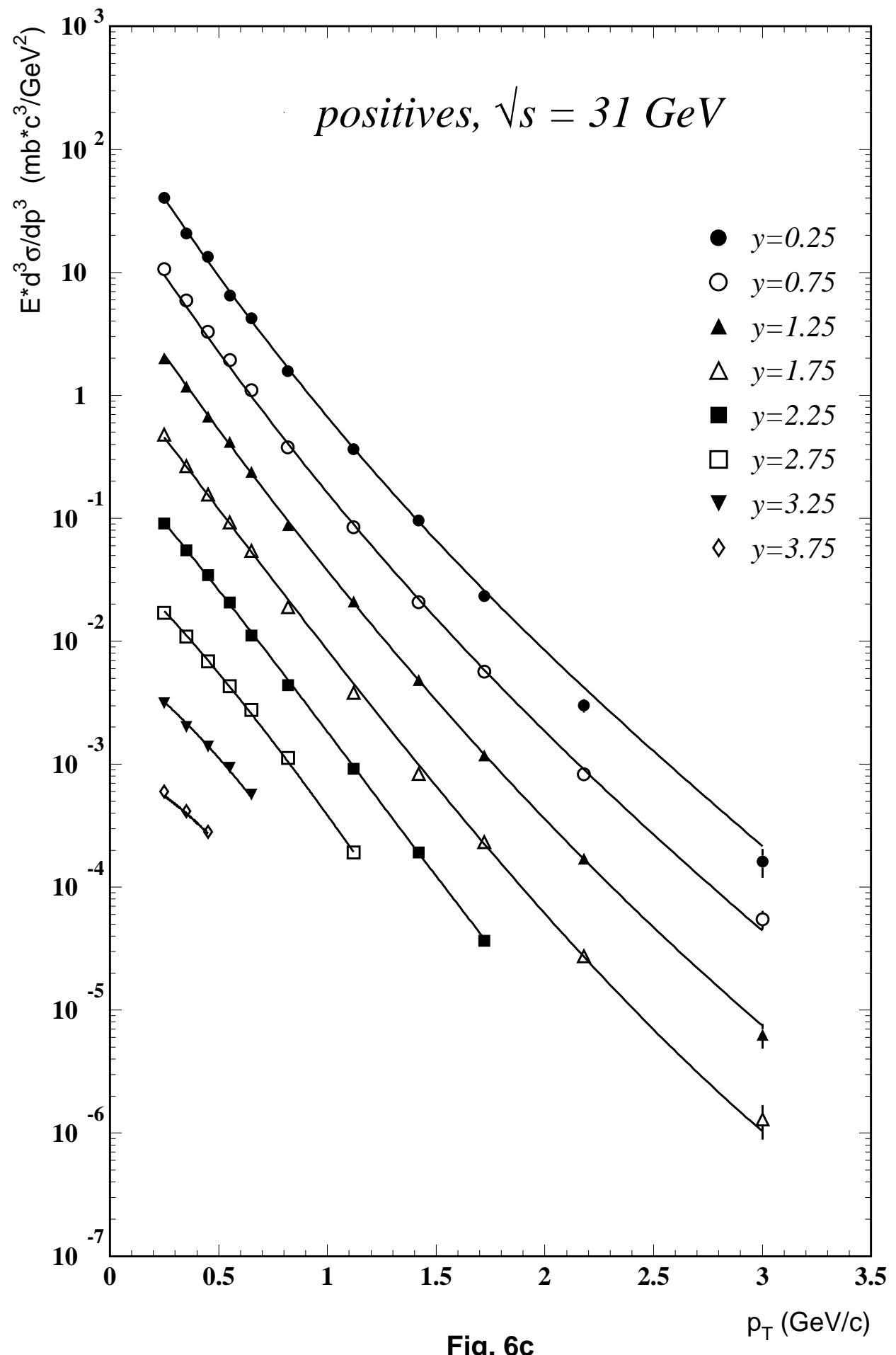

Fig. 6c 


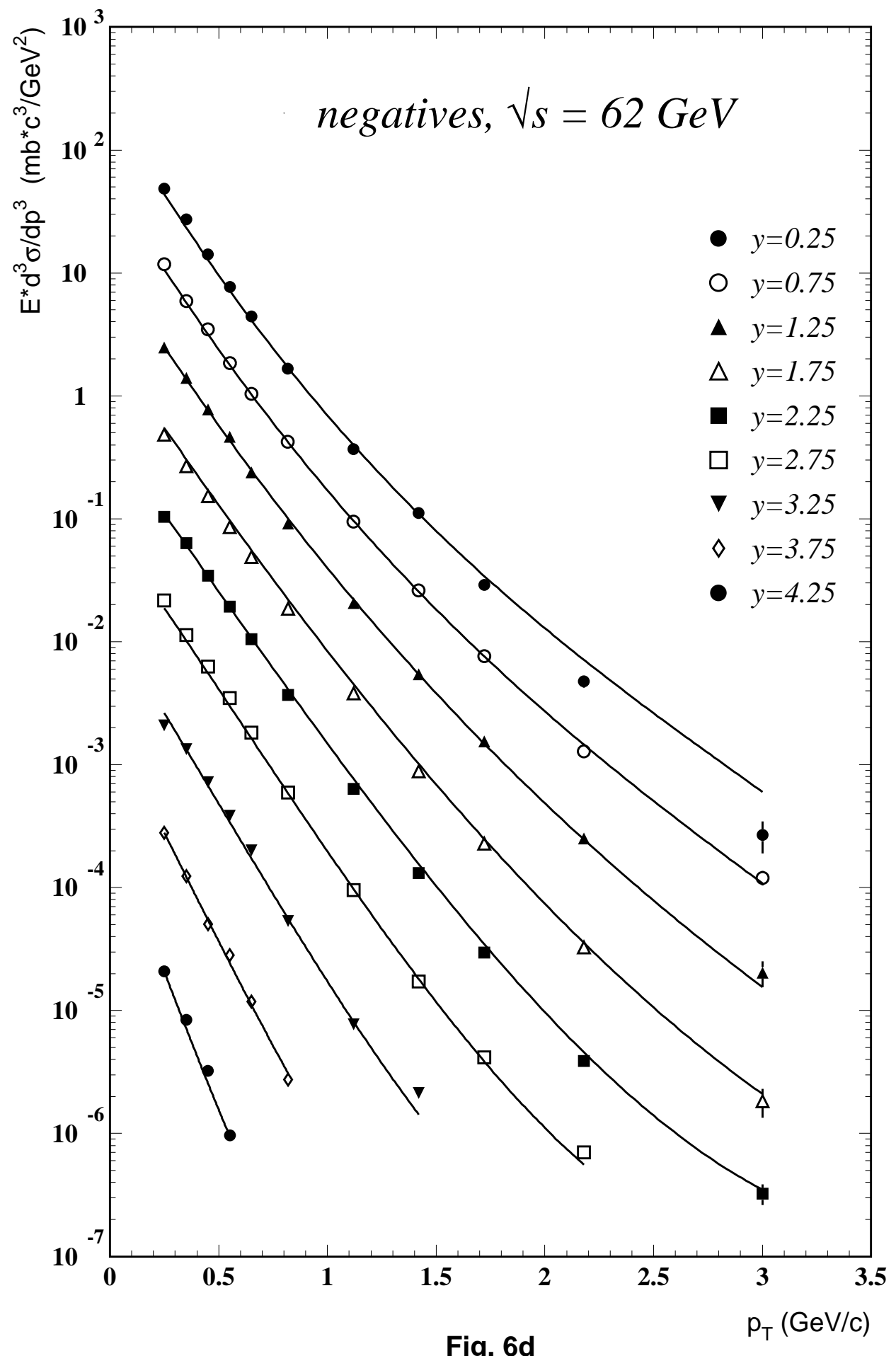

Fig. 6d 


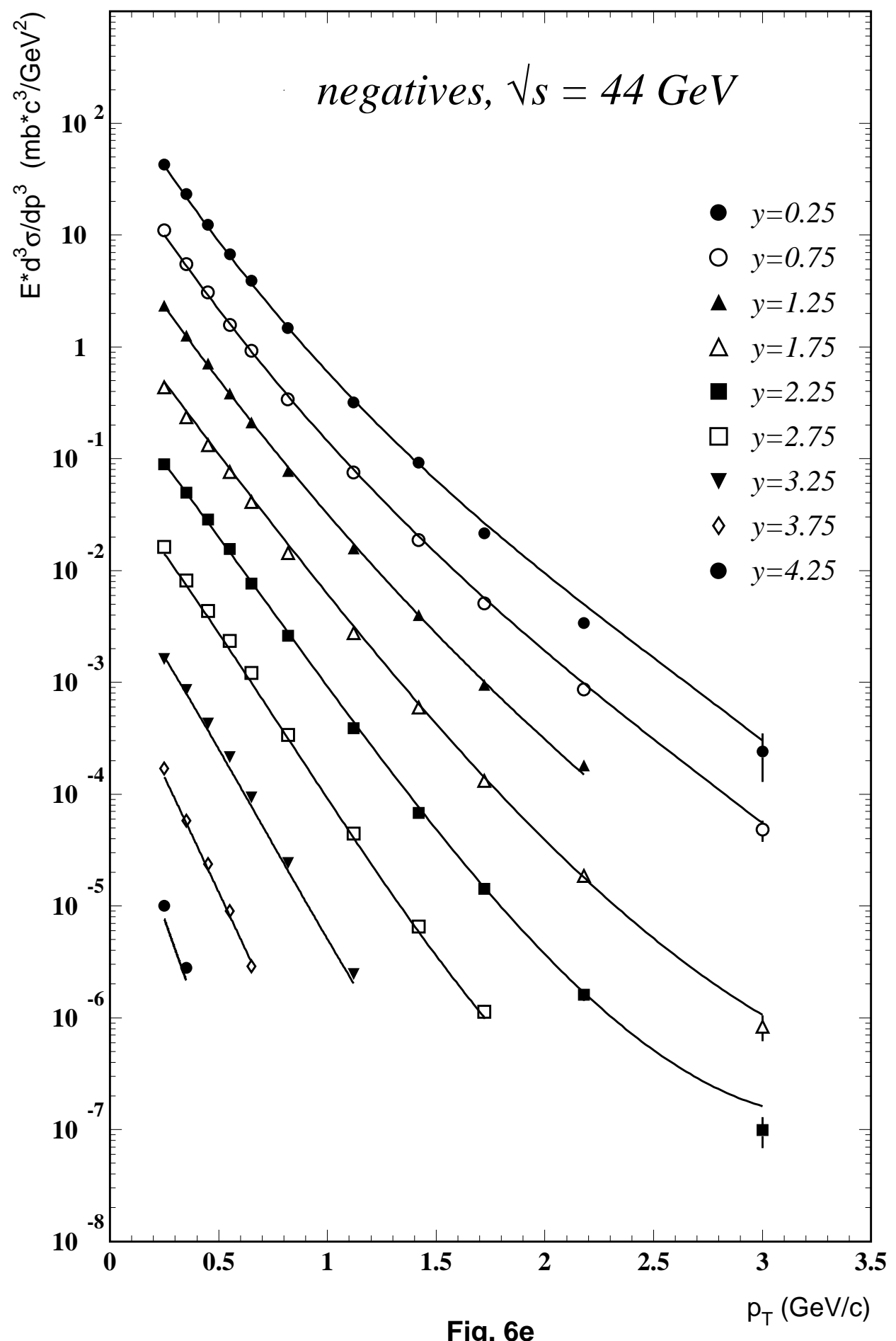

Fig. 6e 


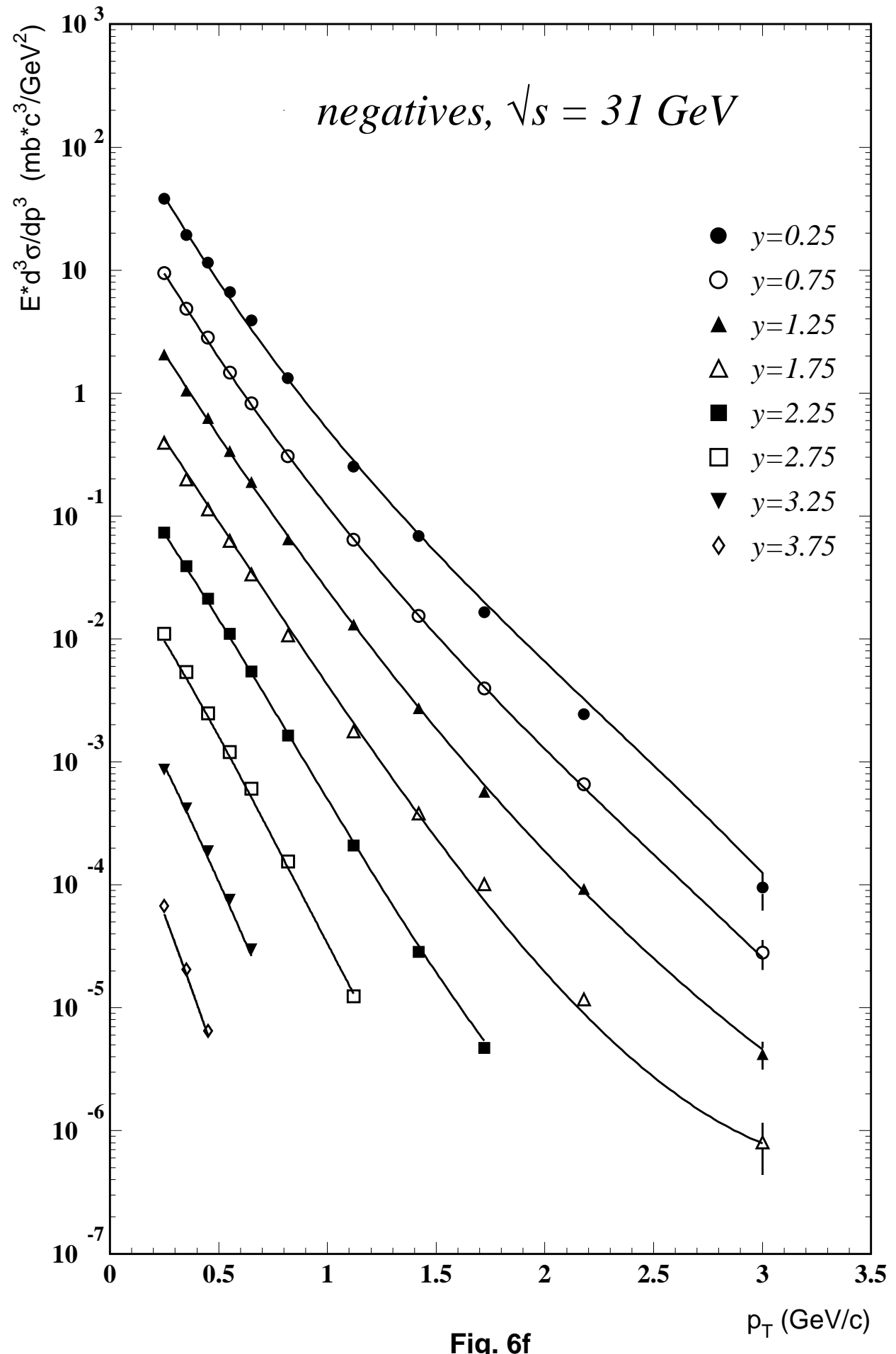

Fig. $6 f$ 